WATPHYS TH-96/20

gr-qc/9612026

\title{
Scalar Wave Falloff in Asymptotically Anti-de Sitter Backgrounds
}

\author{
J. S. F. Chan ${ }^{(1)}$ and R. B. Mann ${ }^{(1,2)}$
}

(1) Department of Applied Mathematics, University of Waterloo, Waterloo, Ontario, Canada N2L 3G1

(2) Department of Physics, University of Waterloo, Waterloo, Ontario, Canada N2L 3G1

PACS numbers: 04.30.Nk, 04.20.Ha, 04.70.-s, 04.25.Dm

\begin{abstract}
Conformally invariant scalar waves in black hole spacetimes which are asymptotically anti-de Sitter are investigated. We consider both the $(2+1)$-dimensional black hole and $(3+1)$-dimensional Schwarzschild-anti-de Sitter spacetime as backgrounds. Analytical and numerical methods show that the waves decay exponentially in the $(2+1)$ dimensional black hole background. However the falloff pattern of the conformal scalar waves in the Schwarzschild-anti-de Sitter background is generally neither exponential nor an inverse power rate, although the approximate falloff of the maximal peak is weakly exponential. We discuss the implications of these results for mass inflation.
\end{abstract}




\section{Introduction}

It is well known that the maximally extended Reissner-Nordström spacetime can be imagined as a collection of different asymptotically flat universes connected by different charged black holes [四]. Except for the Schwarzschild solution, all the special solutions of the more general Kerr-Newman class of spacetimes can have two horizons, the inner and outer horizons. Nevertheless gravitational theorists find that these dualhorizon black holes are unphysical because causality can be violated inside the hole [2]. Moreover any radiation (either electromagnetic or gravitational in nature) that goes into this kind of black hole will be indefinitely blue-shifted at the inner (or Cauchy) horizon [1]. This effect has caused some to expect that this null hypersurface acts like a barricade to the other universes in the maximally extended spacetime.

This infinite blue-shift phenomenon at the Cauchy horizon was first discussed by Penrose in the late sixties [3]. At that time, people believed that any small energy perturbation on these dual-horizon black holes would destroy the Cauchy horizon because the perturbation is indefinitely magnified there, causing an infinite spacetime curvature at the horizon. Thus the null hypersurface would become a spacelike curvature singularity and the gateway to other universes is sealed. Both numerical and analytic approaches [4, 5] suggest that this null hypersurface is perturbatively unstable. However, although singularities are found at the Cauchy horizon, they are not spacetime curvature singularities at all. By taking the diverging stressenergy tensor into account, Hiscock showed that the perturbation only turns the horizon into a so-called whimper singularity: all curvature scalars are finite but a freely falling observer crossing the horizon measures an infinite energy density [6]. This kind of singularity is too mild to seal off the passage to other universes, and so resolution of the issue would necessitate a study that did not rely on perturbation theory.

Poisson and Israel made a breakthrough in this problem by showing that the Cauchy horizon can turn into a scalar spacetime curvature singularity [7]. Unlike Hiscock who considered a Reissner-Nordström black hole irradiated by a flux of incoming radiation, Poisson and Israel imposed both incoming and outgoing fluxes of radiation on a Reissner-Nordström background. The outgoing flux, even if negligibly small in quantity, makes the inner mass function of the black hole inflate without bound at the Cauchy horizon. More precisely, the inner mass of the black hole diverges at a rate of $\exp (\kappa v) / v^{p}$ near the Cauchy horizon. The factor $1 / v^{p}$ comes from the decay rate of the scattered radiation tail [7, 8, 9], where $p>0$ and $v \rightarrow \infty$ at the Cauchy horizon. Regardless of the values of $p$ and the surface gravity $\kappa>0$, the mass parameter always grows although the exponential rate is attenuated by the decaying effect of the radiation tail. This phenomenon is called mass inflation and expected to seal the inner horizon because the diverging mass parameter induces a scalar curvature singularity at the horizon. This result implies that it is inappropriate to maximally extend any dual-horizon black holes beyond the Cauchy horizon because the spacetime is unstable against energy perturbations there. It is generally believed that such perturbations (in the form of gravitational radiation) always exist in more realistic black holes which does not have perfect spherical or axial symmetry. These are scattered around the black hole forming incoming and outgoing fluxes but they will eventually decay away as a tail of late time radiation [9]. In this way, mass inflation is expected to prevent violation of causality.

In addition to the Reissner-Nordström black hole, the mass inflation phenomenon has been found to take place in other black hole configurations [10, 11, 12, 13, 14, 15, 16]. These configurations are in $1+1$, $2+1$ and $3+1$ dimensions as well as in asymptotically non-flat spacetimes. All of these calculations assumed the inverse power-law decay for late time radiation as an ansatz to obtain the inflating mass function near the Cauchy horizon. Since the mass parameter is attenuated by the decaying effect of the radiation tail, it is important to understand the behaviour of the radiative tail in a spacetime other than Reissner-Nordström class. Mellor and Moss have shown that the radiation from perturbations in a de Sitter background exponentially decreases [17]. Strictly speaking this result has nothing to do with late time falloff because the global geometry extends beyond the cosmological horizon. However it indicates that the radiative falloff behaviour is sensitive to the presence of the cosmological constant. More recent work by Ching et al. [18] demonstrated that under certain circumstances the tail can be something other than the 
simple inverse power-law.

In this paper we will study radiative falloff in spacetimes that are not asymptotically flat. We find that the inverse power-law [9] is not universally true, and that in some asymptotically anti-de Sitter spacetimes, the late time tail decays exponentially. The asymptotically anti-de Sitter backgrounds we will study are the $(2+1)$-dimensional black hole [19] and Schwarzschild-anti-de Sitter spacetime.

The outline of our paper is as follows. In section 2 we review the structure of the $(D+1)$-dimensional scalar wave equation in spherically symmetric spacetimes that are not necessarily asymptotically flat, and discuss our numerical approach towards solving it. In section 3 we verify that our numerical approach correctly reproduces the power-law falloff in asymptotically flat spacetimes, and we cross-check this analytically. In the next two sections we study the falloff behaviour in the $(2+1)$-dimensional (or 3D) black hole background and in $(3+1)$-dimensional Schwarzschild-anti-de Sitter spacetime. Concluding remarks and an appendix round out our work.

\section{The Wave Equation in $D+1$ Dimensions}

We shall study scalar waves in different dimensions, since wave equations for higher-spin fields are of a qualitatively similar structure [20]. The (conformally coupled) scalar wave equation in $D+1$ dimensions is

$$
\nabla^{2} \Psi=\xi R \Psi
$$

where $\xi$ is an arbitrary constant. If $\xi=(D-1) /(4 D)$, this equation is conformally invariant. We simplify the problem by considering only static, spherically symmetric $(D+1)$-dimensional spacetimes with metric

$$
d s^{2}=-N(r) d t^{2}+\frac{d r^{2}}{N(r)}+r^{2} d \Omega_{D-1}^{2}
$$

where $N(r)$ is the lapse function and $d \Omega_{D-1}{ }^{2}$ is the metric of a $(D-1)$-dimensional unit sphere. Generalizing Zerilli's separability technique [20] to $D$ dimensions, we assume

$$
\Psi=r^{(1-D) / 2} \psi(t, r) Y_{l}^{D}
$$

The functions $Y_{l}^{D}$ are the $D$-dimensional spherical harmonics which satisfy the equation

$$
\hat{L}^{2}\left[Y_{l}^{D}\right]=-l(l+D-2) Y_{l}^{D}
$$

The product $-l(l+D-2)$ is the eigenvalue of the operator $\hat{L}^{2}$ which is the angular derivative operator. It is straightforward to show that equation (11) gives

$$
-\partial_{t t} \psi(t, r)+N(r) \partial_{r}\left[N(r) \partial_{r} \psi(t, r)\right]-N(r) V_{e}(r) \psi(t, r)=0
$$

The function $V_{e}(r)$ is defined as

$$
V_{e}(r) \equiv \xi R+\frac{D-1}{2 r} \frac{d}{d r} N(r)+\frac{(D-1)(D-3)}{4 r^{2}}[N(r)-1]+\frac{(2 l+D-3)(2 l+D-1)}{4 r^{2}} .
$$

One can rewrite the wave equation (5) as

$$
\text { - } \partial_{t t} \psi(t, r)+N(r) \mathcal{L}[\psi(t, r)]=0
$$

with the help of a spatial differential operator

$$
\mathcal{L} \equiv \partial_{r}\left[N(r) \partial_{r}\right]-V_{e}(r)
$$


Alternatively, if we introduce

$$
x \equiv \int \frac{d r}{N(r)}
$$

then (5) can be written as

$$
\partial_{t t} \psi(t, r(x))-\partial_{x x} \psi(t, r(x))+V(r(x)) \psi(t, r(x))=0 .
$$

or as

$$
\partial_{u v} \psi(u, v)=-\frac{1}{4} N(r(u, v)) V_{e}(r(u, v)) \psi(u, v)
$$

using null co-ordinates $u=t-x$ and $v=t+x$. The function $V(r)$ (defined as $V(r) \equiv N(r) V_{e}(r)$ ) plays the role of a potential barrier which is induced from the background spacetime geometry. Although the potential when written in terms of the tortoise co-ordinate $x$ can be very complicated, equation (10) has the familiar form of a potential scattering problem.

Equation (10) can be integrated numerically in a straightforward fashion by using finite difference method. First of all the D'Alembert operator $\partial_{t t}-\partial_{x x}$ can be discretized as

$$
\frac{\psi(t-\Delta t, x)-2 \psi(t, x)+\psi(t+\Delta t, x)}{\Delta t^{2}}-\frac{\psi(t, x-\Delta x)-2 \psi(t, x)+\psi(t, x+\Delta x)}{\Delta x^{2}}+O\left(\Delta t^{2}\right)+O\left(\Delta x^{2}\right)
$$

using Taylor's theorem. In order to formulate a well-posed Cauchy problem we need to include the initial conditions, which for simplicity we choose to be

$$
\psi(t=0, x)=0 \quad \text { and } \quad \partial_{t} \psi(t=0, x)=u(x) .
$$

Because the field $\psi$ is initially zero, its subsequent evolution is solely the result of the initial impulse of the field $\partial_{t} \psi$. Discretizing the second condition in (13) yields

$$
\frac{\psi(\Delta t, x)-\psi(-\Delta t, x)}{2 \Delta t}=u(x)+O\left(\Delta t^{2}\right)
$$

where we employ a Gaussian distribution with finite support for $u(x)$. We further define

$$
\begin{aligned}
\psi(m \Delta t, n \Delta x) & \equiv \psi_{m, n} \\
V(n \Delta x) & \equiv V_{n} \\
u(n \Delta x) & \equiv u_{n}
\end{aligned}
$$

where the mesh size has to satisfy the condition $\Delta x>\Delta t$ so that the numerical rate of propagation of data is greater than its analytical counterpart. The discretization of the Cauchy problem above then implies

$$
\begin{aligned}
\psi_{-1, n} & =-\Delta t u_{n} \\
\psi_{0, n} & =0 \\
\psi_{m+1, n} & =\left[2-2 \frac{\Delta t^{2}}{\Delta x^{2}}-\Delta t^{2} V_{n}\right] \psi_{m, n}-\psi_{m-1, n}+\frac{\Delta t^{2}}{\Delta x^{2}}\left[\psi_{m, n-1}+\psi_{m, n+1}\right] .
\end{aligned}
$$

As a result, we can follow the evolution of the field $\psi$ starting from the initial data given at time $t=0$.

In the case where the black hole geometry is asymptotically flat, the tortoise coordinate $x$ goes from negative infinity to positive infinity. Therefore our Cauchy problem is similar to the infinite string problem in which the initial data propagates towards left and right indefinitely. The initial data no longer enjoys this privilege when the background is asymptotically anti-de Sitter because the tortoise coordinate goes from 
minus infinity to zero only. In other words, the right-propagating data cannot travel in this direction forever. Analogous to the semi-infinite vibrating string problem, boundary conditions at spatial infinity (i.e. $x=0$ ) are needed in the asymptotically anti-de Sitter background in order to formulate the problem appropriately. Two types of boundary conditions that are widely used in anti-de Sitter backgrounds are the Dirichlet and Neumann conditions [21]. In our case, the former reads

$$
\psi(t, x=0)=0 \quad \text { and } \quad \partial_{x} \psi(t, x=0)=1
$$

whilst the latter is simply

$$
\psi(t, x=0)=1 \quad \text { and } \quad \partial_{x} \psi(t, x=0)=0 .
$$

We shall consider employing both of these boundary conditions at spatial infinity for our numerical computations whenever the background geometry is asymptotically anti-de Sitter.

\section{Asymptotically Flat Backgrounds}

In this section we will review the behaviour of radiative falloff in asymptotically flat background spacetimes [9]. We will present the results of the numerical calculation first.

Figure 11 shows a sample inverse power-decay of a scalar wave in the Schwarzschild background spacetime of mass $M$. We solve the wave equation (10) numerically using the scheme discussed in the previous section. The compact initial Gaussian impulse is centered at a distance $r=10 M$ (or $x=12.76 M$ ) and for simplicity we choose the $l=1$ spherical harmonic. Figure 1 shows how the magnitude of the scalar field $\psi$ at a distance $r=20 M$ (i.e. $x=24.40 M$ ) evolves. Using linear regression, we find that the slope of the straight line on the graph is -5.026 in agreement with the analytic prediction of an inverse power-law falloff with exponent $2 l+3[9]$.

Figures 2 to 4 show the same potential barrier $V(x)$ that is responsible for this falloff behaviour. Figure 3 shows an exponential decrease of the left side of the potential function; this is a result of the fact that the event horizon is located at $x=-\infty$. On the other hand, the power-law decrease on the right side of $V(x)$ shown in figure 1 is a direct consequence of the use of an asymptotically flat background. If we compare figures 2 to 1 with figures 5 to 7 which represent the potential function in Schwarzschild-de Sitter (SdS) spacetime, we find that the right side of $V(x)$ has different decaying behaviour even the overall appearance of $V(x)$ on the linear graph is very similar. It is this difference that distinguishes the falloff behaviour in the two backgrounds

Now let us consider a scalar wave in an asymptotically flat $(D+1)$-dimensional background. For the remainder of this section we will restrict our attention to the case where the number of spatial dimensions $D$ is odd. The motivation for this may be traced back to Huygen's Principle, which implies that in even spatial dimensions the scalar wave obeying the equation $\nabla^{2} \Psi=0$ always develops a tail, regardless of whether or not the asymptotically flat background is sourceless. Consequently identification of the tail part of the wave that is due to solely to backscattering becomes quite problematic when $D$ is even.

Inspired by the work of Ching et. al. [18, 22], we consider a spherically symmetric static metric of the form (2) with lapse function

$$
N(r)=1-m \frac{(\ln |r|)^{\beta}}{r^{\alpha}} .
$$

The constants $\alpha$ and $\beta$ are integers, where $\beta \geq 0$ but $\alpha>0$. The other constant $m$ is a real number. When we have $\alpha=1, \beta=0$ and $D=3$, this becomes a Schwarzschild background of mass $m / 2$. Once we have 


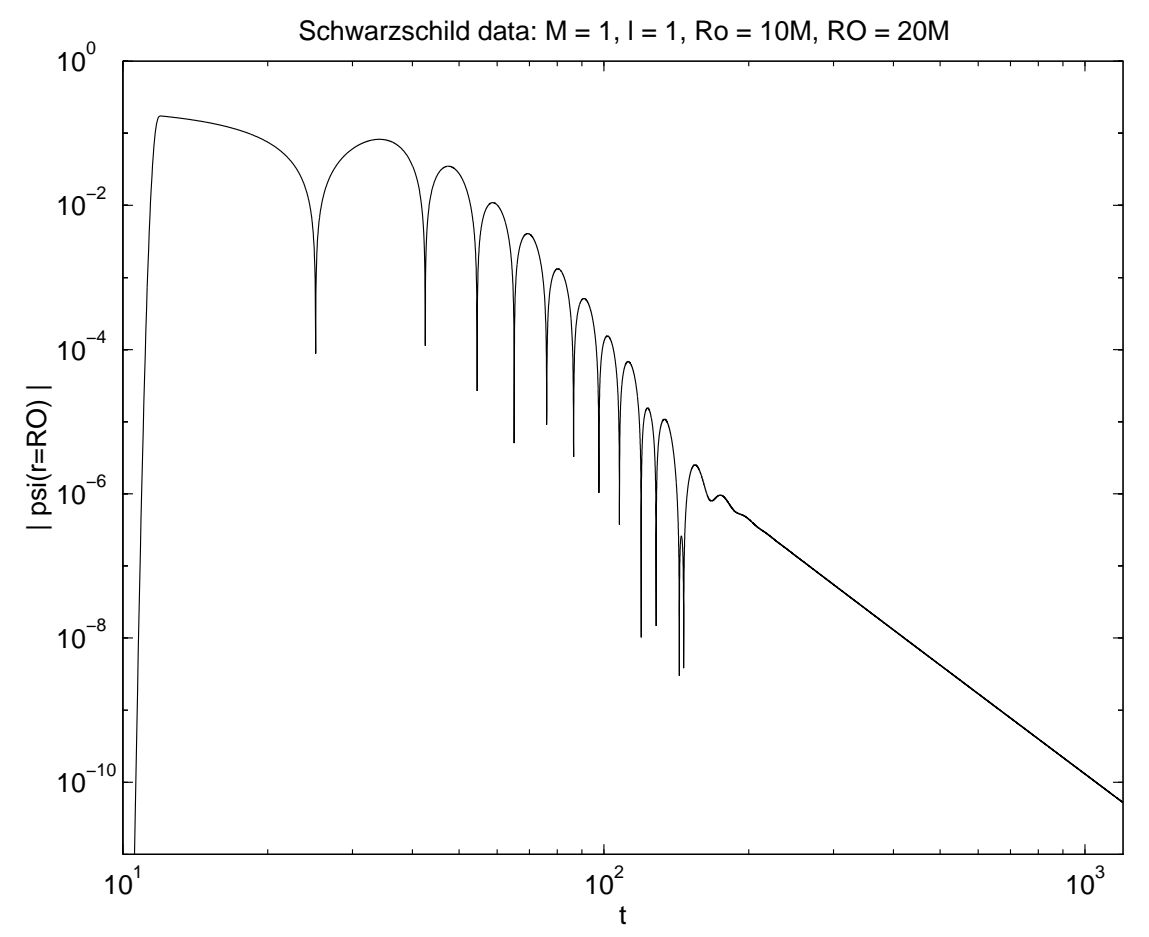

Figure 1: The decay of a scalar wave in Schwarzschild background. Prior to $t=200$ the decay is accompanied by 'ringing' of the quasi-normal modes, after which the falloff rate is that of an inverse power-law.

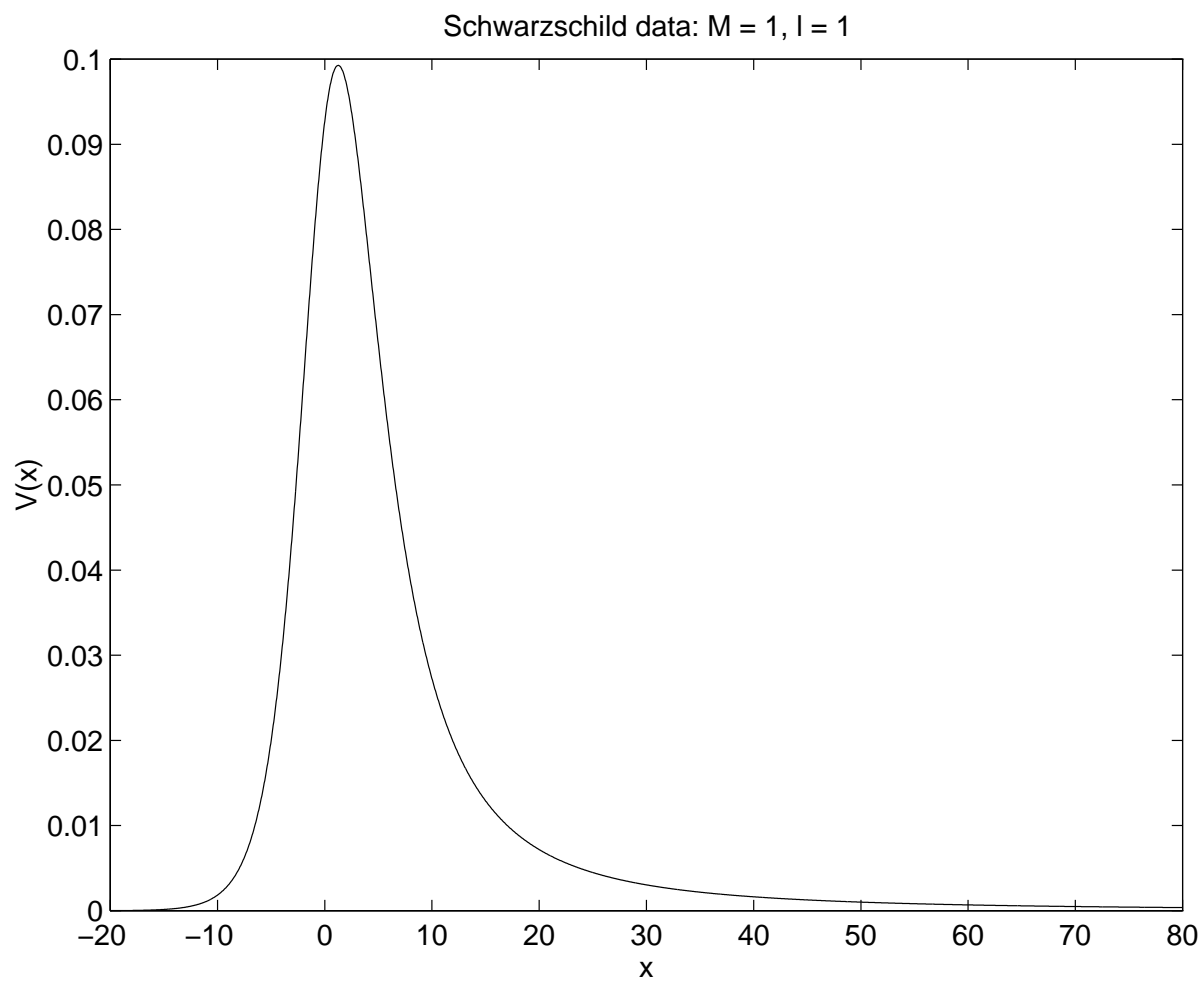

Figure 2: Potential barrier for the Schwarzschild background 


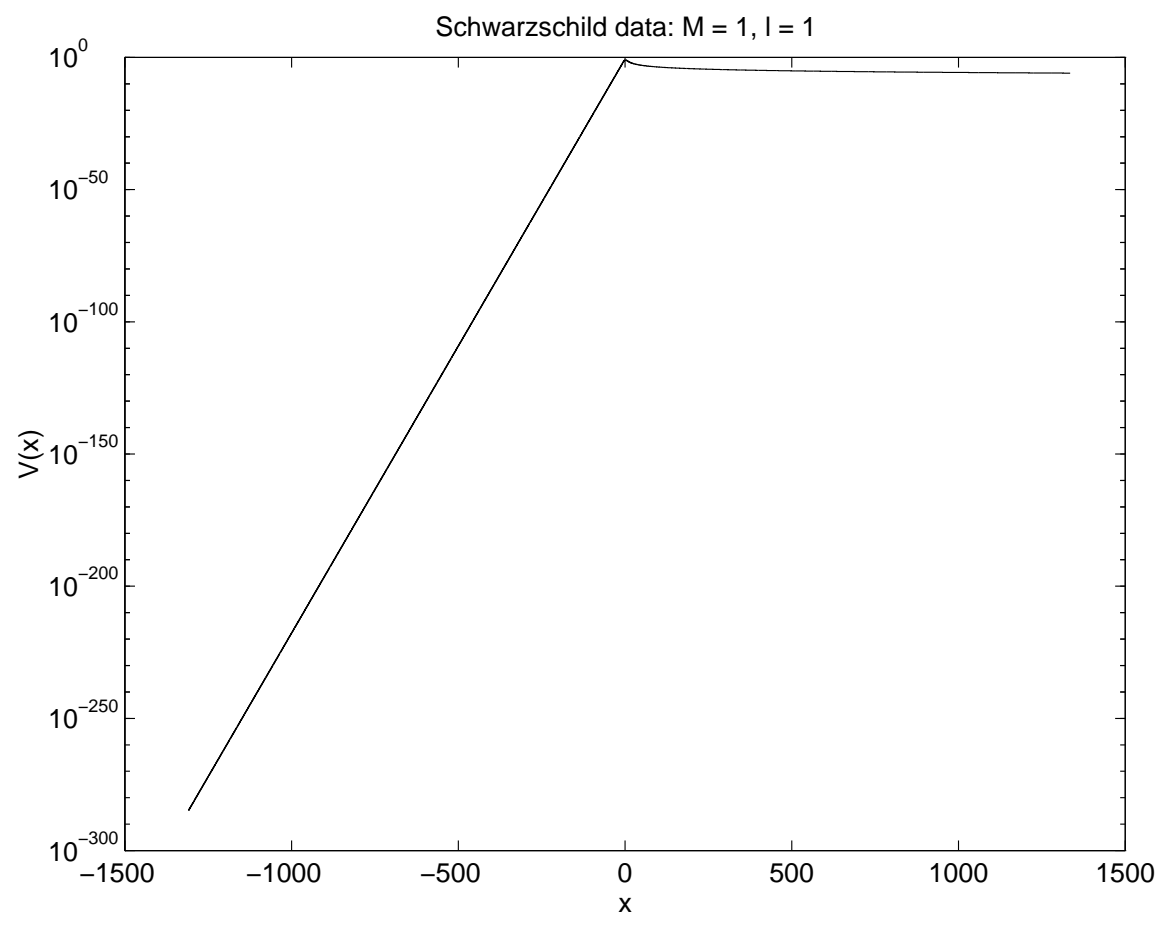

Figure 3: Exponential decrease on the left side of the barrier in $V(x)$

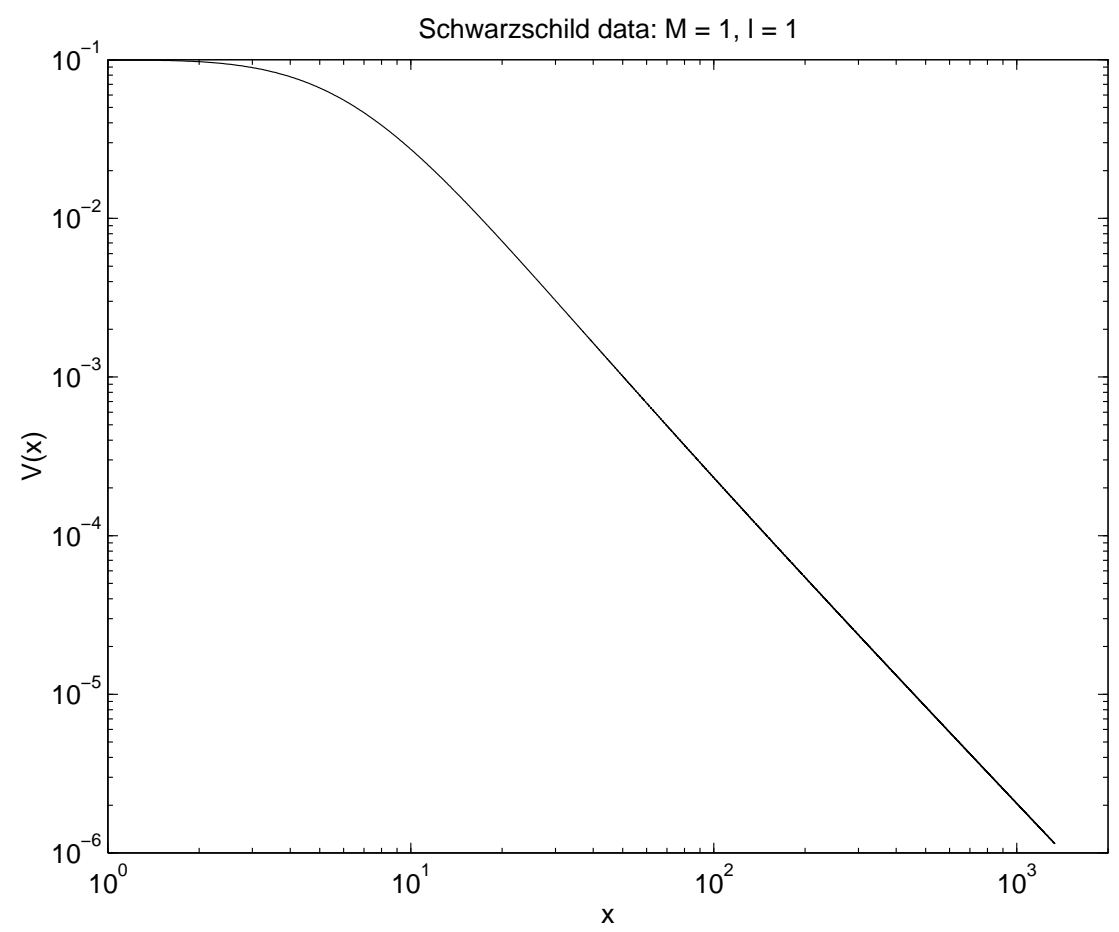

Figure 4: Inverse power decrease on the right side of the barrier of $V(x)$ 


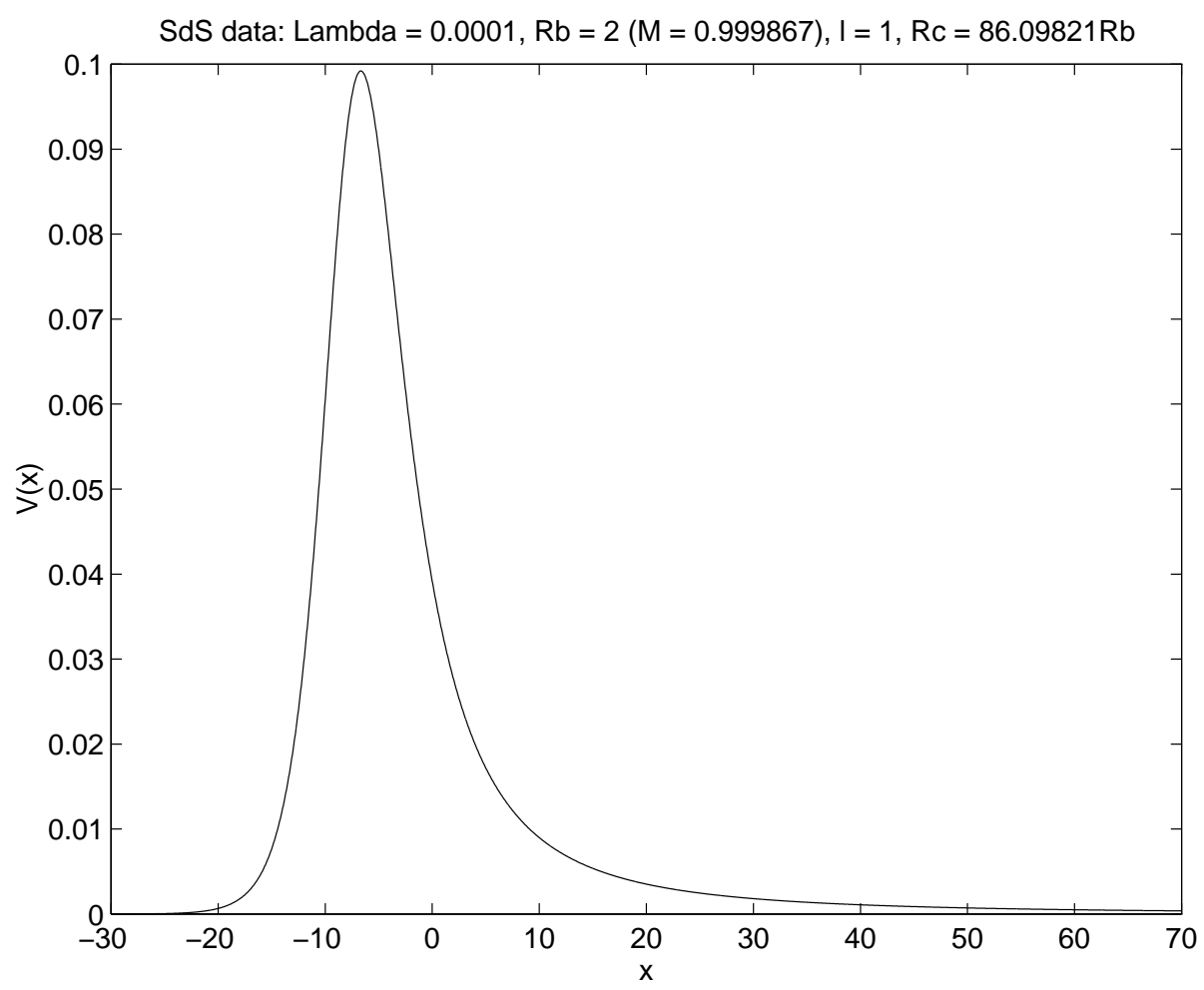

Figure 5: Potential barrier for the Schwarzschild-de Sitter background

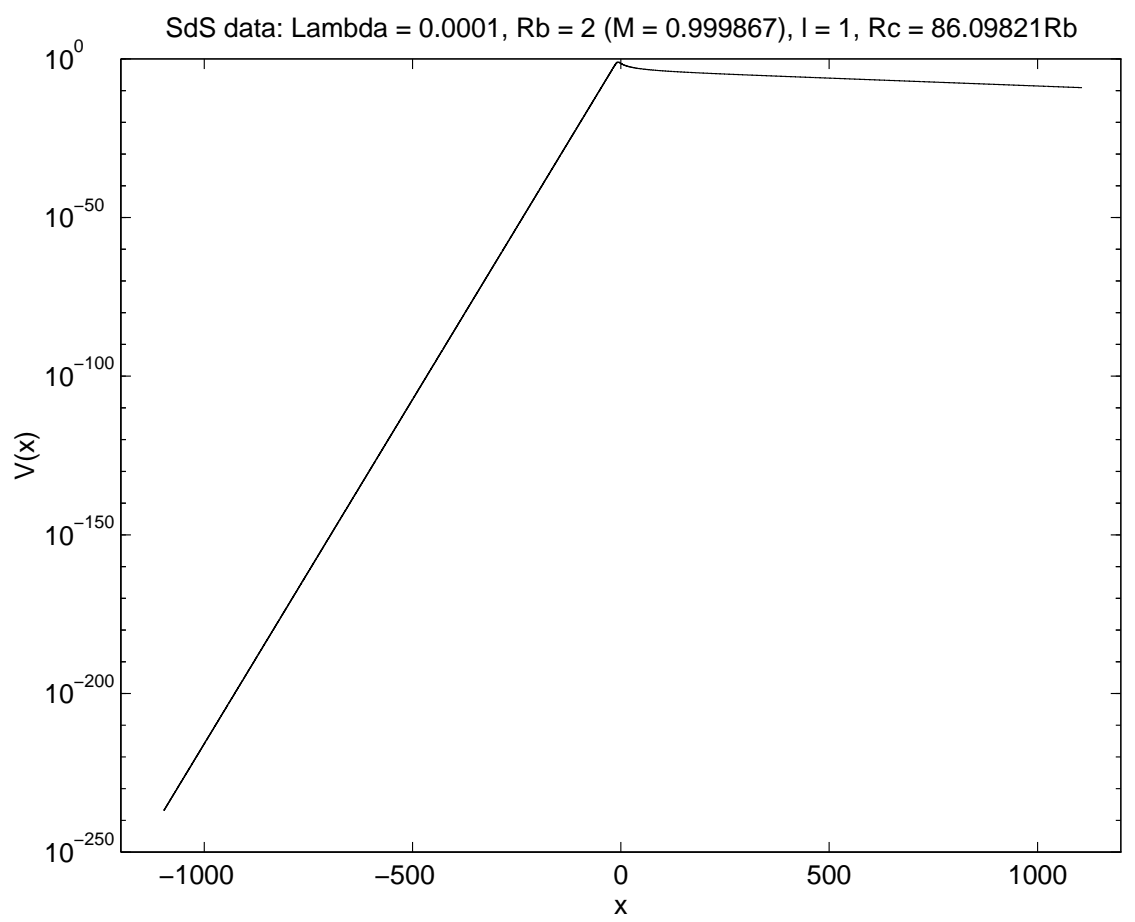

Figure 6: Exponential decreasing nature on the left side of $V(x)$ in SdS background 


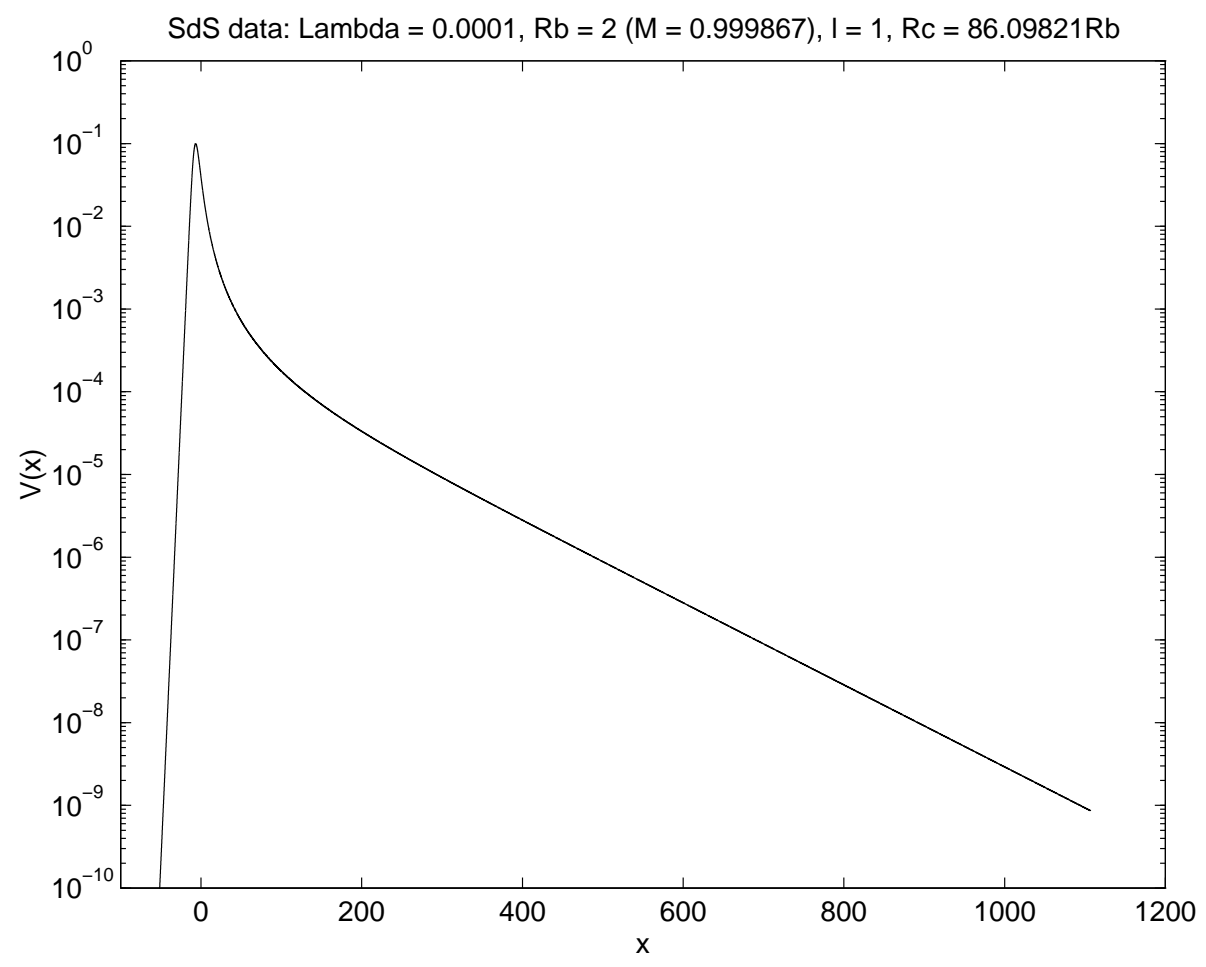

Figure 7: Exponential decrease of the right side of $V(x)$ in SdS background

the lapse function, we can compute the potential function

$$
\begin{aligned}
V_{e}(r)= & \frac{(2 l+D-3)(2 l+D-1)}{4 r^{2}}+m \frac{4 \xi(D-1-\alpha)(D-2-\alpha)-(D-1)(D-3-2 \alpha)}{4} \frac{(\ln |r|)^{\beta}}{r^{\alpha+2}} \\
& +m \beta \frac{2 \xi(2 D-3-2 \alpha)-D+1}{2} \frac{(\ln |r|)^{\beta-1}}{r^{\alpha+2}}+m \beta(\beta-1) \xi \frac{(\ln |r|)^{\beta-2}}{r^{\alpha+2}}
\end{aligned}
$$

from (可).

We first find the static solution $\psi_{S}(r)$ of wave equation (可), by obtaining the solution of the equation $\mathcal{L}\left[\psi_{S}(r)\right]=0$. It is straightforward to show that $\psi_{S}(r)$ has the following form:

$$
\psi_{S}(r)=r^{-\gamma} \sum_{j=0}^{\infty} \frac{a_{j}(r)}{r^{j \alpha}}+r^{\gamma+1} \sum_{j=0}^{\infty} \frac{c_{j}(r)}{r^{j \alpha}},
$$

where $\gamma \equiv l+(D-3) / 2$. Notice that $\gamma$ is an integer when the spatial dimension $D$ is odd. Except when $D=1$ this integer is always positive. Hence it is the first sum of the solution that is physically relevant since it vanishes for large $r$, and we choose this as the static solution. For the remainder of this section, we assume that $D \geq 3$ so that this choice of $\psi_{S}(r)$ is valid. The coefficients $a_{0}(r)$ and $c_{0}(r)$ are arbitrary constants but the other coefficients are all polynomial in $\ln |r|$. The generating equations for $a_{j}(r)$ and $c_{j}(r)$ are given in Appendix A.

We follow the approach in refs. [9, 23] and let

$$
\psi_{I}=\sum_{i=0}^{\infty} B_{i}(r)\left[g^{(-i)}(u)+(-1)^{i} f^{(-i)}(v)\right]
$$

be the form of the initial wave, i.e. the wave emitted by a star at the onset of gravitational collapse. In other words, this is the time when $t \ll r$. The functions $g(u)$ and $f(v)$ are as yet unknown. The term $g^{(-i)}(u)$ 
represents $i$ integrations of the function $g(u)$ with respect to $u$; similarly for $f^{(-i)}(v)$. Using (26), equation (11) becomes

$$
\begin{aligned}
0= & \frac{1}{2} N(r) \frac{d}{d r} B_{0}(r)\left[g^{(1)}(u)-f^{(1)}(v)\right] \\
& -\frac{1}{4} N(r) \sum_{i=0}^{\infty}\left\{\mathcal{L}\left[B_{i}(r)\right]-2 \frac{d}{d r} B_{i+1}(r)\right\}\left[g^{(-i)}(u)+(-1)^{i} f^{(-i)}(v)\right] .
\end{aligned}
$$

This equation has a set of solutions

$$
\begin{aligned}
B_{0}(r) & =1 \\
B_{i+1}(r) & =\frac{1}{2} N(r) \frac{d}{d r} B_{i}(r)-\frac{1}{2} \int V_{e}(r) B_{i}(r) d r, \quad i=0,1,2, \cdots
\end{aligned}
$$

where we have set $B_{0}(r)=$ constant $=1$ without loss of generality. The pair of equations above allow us to generate $B_{i}(r)$ hierarchically in a straightforward manner.

We can split each $B_{i}(r)$ into two parts, denoted by $B_{i}^{P}(r)$ and $B_{i}^{T}(r) . \quad B_{i}^{P}(r)$ is defined as the $m$ independent portion of $B_{i}(r)$, while $B_{i}^{T}(r)$ is the rest which is $m$-dependent. Physically, the part $B_{i}^{P}(r)$ represents the wave on the lightcone because it is the part that would be generated if the background were flat $(m=0)$. Price referred to this part as the primary wave which depends only on the mode of the spherical harmonics. The other part $B_{i}^{T}(r)$ is called the tail of the wave because it is created by the presence of the spacetime curvature and is off the lightcone due to scattering. Given equations (28) and (29), one can show that the primary part of $B_{i}(r)$ is simply

$$
B_{i}^{P}(r)=\frac{\Gamma(\gamma+1+i)}{2^{i} i ! \Gamma(\gamma+1-i) r^{i}} .
$$

When $D$ is odd, $\gamma$ is an integer and the sequence $\left\{B_{i}^{P}(r)\right\}_{i=0}$ truncates. However if $D$ is even, the sequence does not terminate and the primary part of the initial wave $\psi_{I}$ has infinitely many terms. Indeed when this is the case, it is inappropriate to call $B_{i}^{P}(r)$ the primary part because there are tails present. The approach breaks down because the primary and tail parts of the wave become indistinguishable. This is the reason we restrict ourselves to odd $D$ in this section as mentioned earlier.

Let us now consider the tail part of $B_{i}(r)$. Unlike the primary part, $B_{i}^{T}(r)$ has no simple solution. Fortunately one can always generate $B_{i}(r)$ recursively. Note that $B_{i}^{T}(r)$ is of order $O\left((\ln |r|)^{\beta} / r^{i+\alpha}\right)$.

Since $\gamma$ is an integer in odd spatial dimensions, it is convenient to define the functions $G(u)$ and $F(v)$ as

$$
G(u)=g^{(-\gamma)}(u) \quad \text { and } \quad F(v)=f^{(-\gamma)}(v)
$$

such that the initial wave can be expressed as $\psi_{I}=\sum B_{i}(r) T_{I}^{\gamma-i}(u, v)$ where

$$
T_{I}^{\gamma-i}(u, v) \equiv G^{(\gamma-i)}(u)+(-1)^{i} F^{(\gamma-i)}(v) .
$$

When the background is flat $(m=0)$, only the first $\gamma+1$ terms of $B_{i}^{P}(r)$ survive. We expect that any outgoing radiation will propagate to spatial infinity without any scattering in a flat background. Therefore we must have

$$
F^{(\gamma)}(v)=F^{(\gamma-1)}(v)=\cdots=F^{(1)}(v)=F^{(0)}(v)=0
$$

because $F(v)$ represents the scattered infalling radiation and the $B_{i}^{P}(r)$ are non-zero only for $0 \leq i \leq \gamma$. In other words, we insist that $F(v)=0$. 
For concreteness, we suppose the scalar wave starts leaving the star at retarded time $u=U_{0}$; that is to say the scalar field is zero before $u=U_{0}$. Continuity then implies

$$
\begin{array}{r}
G^{(\gamma)}\left(U_{0}\right)=G^{(\gamma-1)}\left(U_{0}\right)=\cdots=G^{(1)}\left(U_{0}\right)=G^{(0)}\left(U_{0}\right)=0 \\
G^{(-1)}(u)=\int_{U_{0}}^{u} G^{(0)}(\zeta) d \zeta, \quad G^{(-2)}(u)=\int_{U_{0}}^{u} G^{(-1)}(\zeta) d \zeta,
\end{array}
$$

We also assume that there is essentially no emission from the star after $u=U_{1}>U_{0}$ (due to gravitational red shift). When the star possesses no initial static moment at the onset of collapse, this cutoff condition is translated into

$$
G^{(\gamma)}(u)=G^{(\gamma-1)}(u)=\cdots=G^{(1)}(u)=G^{(0)}(u)=0, \quad \forall u \geq U_{1}>U_{0}
$$

because only the terms $G(u)$ to $G^{(\gamma)}(u)$ which represent the primary wave correspond to the emission from the star. This condition implies

$$
G^{(-1)}(u)=\int_{U_{0}}^{u} G^{(0)}(\zeta) d \zeta=\int_{U_{0}}^{U_{1}} G^{(0)}(\zeta) d \zeta=\text { constant }, \quad \forall u \geq U_{1} .
$$

Therefore if there is no static moment before $u=U_{0}$, the scalar wave after time $u=U_{1}$ is

$$
\psi_{u \geq U_{1}}=\sum_{i=\gamma+1}^{\infty} B_{i}(r) T_{I}^{\gamma-i}(u, v)
$$

where $T_{I}^{-1}(u, v)$ is only a constant and $B_{i}(r)=B_{i}^{T}(r)$ is of order $O\left((\ln |r|)^{\beta} / r^{i+\alpha}\right)$. So the first term of this solution which is time-independent is of order $O\left((\ln |r|)^{\beta} / r^{\gamma+\alpha+1}\right)$. Physically, this says the tail of the perturbation persists after the time $u=U_{1}$. The primary part of the wave has been "washed out" by the gravitational red shift before $u=U_{1}$.

The situation is more complicated if the star carries a static moment at the onset of the collapse because the static wave $\psi_{S}(r)$ can also be divided into primary and tail parts. In this case, we superimpose $\psi_{S}(r)$ of (25) and $\psi_{I}(r)$ of (26) together to form a new initial scalar wave. We also suppose that only the tail of the perturbation persists after the retarded time $u=U_{1}[9]$. For the superimposed initial wave this cutoff condition requires

$$
G^{(0)}(u)=-\frac{2^{\gamma} \gamma !}{(2 \gamma) !}, \quad \forall u \geq U_{1} \geq U_{0}
$$

which yields

$$
G^{(\gamma)}(u)=G^{(\gamma-1)}(u)=\cdots=G^{(1)}(u)=0
$$

The restriction on $G(u)$ above ensures that the primary part of (26) can be cancelled by the $m$-independent part of (25) properly after the time $U_{1}$, leaving the combined $\psi_{I}$ to be $m$-dependent only after $U_{1}$. As a result, the wave with initial static moment will become

$$
\psi_{u \geq U_{1}}=\frac{1}{r^{\gamma}} \sum_{j=1}^{\infty} \frac{a_{j}(r)}{r^{j \alpha}}+\sum_{i=\gamma}^{\infty} B_{i}^{T}(r) T_{I}^{\gamma-i}(u, v) .
$$

One can show that the first sum in the equation above is of order $O\left((\ln |r|)^{\beta} / r^{\gamma+\alpha}\right)$ because the coefficient function $a_{1}(r)$ is a polynomial in $\ln |r|$ with degree $\beta$. In the second sum, the term $T_{I}^{0}(u, v)$ is a constant which equals to $G(u)$, and $B_{\gamma}^{T}(r)$ is of order $O\left((\ln |r|)^{\beta} / r^{\gamma+\alpha}\right)$. Therefore instead of having a time independent part of order $O\left((\ln |r|)^{\beta} / r^{\gamma+\alpha+1}\right)$, the wave with initial static moment has an order $O\left((\ln |r|)^{\beta} / r^{\gamma+\alpha}\right)$ only. 
Now we turn our attention to the scalar wave at late time. By late time, we mean $t \gg r$. For the late time wave $\psi_{L}$, we introduce another ansatz

$$
\psi_{L}=\sum_{i=0}^{\infty} C_{i}(r) T_{L}^{i}(u, v), \quad T_{L}^{i}(u, v) \equiv I^{(i)}(u)+(-1)^{i} H^{(i)}(v) .
$$

By substituting $\psi=\psi_{L}$, equation (11) becomes

$$
\frac{1}{4} N(r) \mathcal{L}\left[C_{0}(r)\right] T_{L}^{0}(u, v)+\frac{1}{4} N(r) \sum_{i=0}^{\infty}\left\{\mathcal{L}\left[C_{i+1}(r)\right]-2 \frac{d}{d r} C_{i}(r)\right\} T_{L}^{i+1}(u, v)=0
$$

This equation has another set of solutions

$$
\begin{aligned}
\mathcal{L}\left[C_{0}(r)\right] & =0, \\
\mathcal{L}\left[C_{i+1}(r)\right] & =2 \frac{d}{d r} C_{i}(r), \quad i=0,1,2, \cdots
\end{aligned}
$$

Unlike the case for the initial wave $\psi_{I}$, (for which the functions $B_{i}(r)$ can be calculated recursively using straightforward differentiation and integration), recursive generation of the functions $C_{i}(r)$ involve inverting the differential operator $\mathcal{L}$. The zero order equation has a solution

$$
C_{0}(r)=r^{\gamma+1} \sum_{j=0}^{\infty} \frac{c_{j}(r)}{r^{j \alpha}}
$$

The coefficient functions $c_{j}(r)$ are those in equations (25). They can be calculated by using the generating equations in Appendix A. The other inhomogeneous differential equation (45) has a solution of the form

$$
C_{i}(r)=r^{\gamma+1+i} \sum_{j=0}^{\infty} \frac{c_{j}^{i}(r)}{r^{j \alpha}}
$$

where $i=0,1,2, \cdots$ When $i=0$, it is understood that $c_{j}^{0}(r)=c_{j}(r)$. The coefficients $c_{j}^{i}(r)$ are also given in Appendix A. Since each coefficient $c_{0}^{i}(r)$ is a constant instead of a polynomial in $\ln |r|$, we can estimate the order of the late time wave $\psi_{L}$ as

$$
\psi_{L}=\sum_{i=0}^{\infty} O\left(r^{\gamma+1+i}\right) T_{L}^{i}(u, v)
$$

Finally we match the late time solution to the initial solution at some transient period where $u, v$ and $r$ are of the same order. As the background is asymptotically flat, the tortoise coordinate $x$ must have an order similar to that of $r$ in this transient region. That is to say the orders of $r$ and $t$ are the same. The initial wave $\psi_{u \geq U_{1}}$ in this period becomes

$$
\begin{array}{ll}
\psi_{u \geq U_{1}} \sim O\left(\frac{(\ln |r|)^{\beta}}{r^{\gamma+\alpha+1}}\right), & \text { without initial static moment } \\
\psi_{u \geq U_{1}} \sim O\left(\frac{(\ln |r|)^{\beta}}{r^{\gamma+\alpha}}\right), & \text { with initial static moment }
\end{array}
$$

In order to have consistent orders in the transient period, we must have

$$
T_{L}^{0}(t, t) \sim O\left(\frac{(\ln |t|)^{\beta}}{t^{2 \gamma+2+\alpha}}\right)
$$


if the star has no initial static moment; whilst the order of $T_{L}^{0}$ must be

$$
T_{L}^{0}(t, t) \sim O\left(\frac{(\ln |t|)^{\beta}}{t^{2 \gamma+1+\alpha}}\right)
$$

if the star has a static moment at the onset of collapse. In other words equations (49) and (50) give the falloff behaviour of the wave at late time. The inverse power falloff behaviour modified by a logarithmic term was first noted by Ching et al. 22]. For a Schwarzschild background, we set $D=3, \alpha=1$ and $\beta=0$ and have $\gamma=l$, yielding the familiar power-law decay rate [9, 23].

\section{The 3D Black Hole Background}

The $(2+1)$-dimensional black hole spacetime obtained by Banados Teitelboim and Zanelli 19 is a spacetime which satisfies the vacuum Einstein equations with a negative cosmological constant $(\Lambda<0)$. Its metric is

$$
\begin{aligned}
d s^{2} & =-N(r) d t^{2}+\frac{d r^{2}}{N(r)}+r^{2}\left(-\frac{J}{2 r^{2}} d t+d \phi\right)^{2}, \\
N(r) & =|\Lambda| r^{2}-M+\frac{J^{2}}{4 r^{2}}
\end{aligned}
$$

and is actually anti-de Sitter spacetime with identifications. The constant $M>0$ is the quasi-local mass of the black hole and $J$ is the angular momentum of the hole [24]. For our purposes we shall regard the above metric as a spacetime which is asymptotically anti-de Sitter.

When $J=0$, the wave equation (1) gives (10) with

$$
V_{e}(r)=\frac{3|\Lambda|}{4}(1-8 \xi)+\frac{M+4 l^{2}}{4 r^{2}}
$$

In this case, the tortoise coordinate $x$ is given by

$$
x \equiv \int \frac{d r}{N(r)}=\frac{1}{2 \sqrt{|\Lambda| M}} \ln \left|\frac{\sqrt{|\Lambda|} r-\sqrt{M}}{\sqrt{|\Lambda|} r+\sqrt{M}}\right| .
$$

As $r$ goes from $\sqrt{M /|\Lambda|}$ to infinity, the tortoise coordinate has a range $(-\infty, 0)$. Thus we can write $r$ in terms of $x$ as

$$
r=\sqrt{\frac{M}{|\Lambda|}} \frac{1+\exp (2 \sqrt{|\Lambda| M} x)}{1-\exp (2 \sqrt{|\Lambda| M} x)}
$$

For conformal wave in $2+1$ dimensions, the parameter $\xi$ equals $1 / 8$ and the potential barrier $V$ becomes

$$
V(x)=V_{0} \frac{\exp (2 \lambda x)}{(1+\exp (2 \lambda x))^{2}} \approx V_{0} \exp (2 \lambda x),
$$

where, for convenience, we have defined

$$
V_{0} \equiv|\Lambda|\left(M+4 l^{2}\right)>0 \quad \text { and } \quad \lambda \equiv \sqrt{|\Lambda| M}>0
$$

The procedure for finding a solution to the conformal scalar wave equation is as follows. We first write down the representation of the solution $\psi$ in terms of Green's functions. The rest of the problem then reduces 
to that of looking for the correct Green's function. We will Fourier transform from the time domain to the frequency domain and obtain the Green's function in frequency space. Once we have the Green's function in frequency domain, an inverse Fourier transformation will yield the solution $\psi(t, x)$. The solution is exact for the approximate potential (56). We will then use this exact solution and the Born approximation to compute the next correction of the solution for a more accurate description of the potential. A case when $J \neq 0$ will be studied as well.

We first assume that there exists a Green's function

$$
G(x, \xi ; t-\tau)=G(\xi, x ; t-\tau)
$$

which is zero when $t<\tau$. We define an operator $D$ as

$$
D=\partial_{t t}-\partial_{x x}+V(x)
$$

such that the Green's function with respect to this operator has the property

$$
D G(x, \xi ; t-\tau)=\left[\partial_{t t}-\partial_{x x}+V(x)\right] G(x, \xi ; t-\tau)=\delta(t-\tau) \delta(x-\xi) .
$$

The inner product between $D \psi(t, x)$ and $G(x, \xi ; t-\tau)$ gives

$$
\begin{aligned}
\psi(t, x)= & \int_{-\infty}^{0}\left[G(x, \xi ; t) \partial_{t} \psi(0, \xi)+\psi(0, \xi) \partial_{t} G(x, \xi ; t)\right] d \xi \\
& +\int_{0}^{\infty}\left[G(x, \xi ; t-\tau) \partial_{\xi} \psi(\tau, \xi)-\psi(\tau, \xi) \partial_{\xi} G(x, \xi ; t-\tau)\right]_{\xi=-\infty}^{\xi=0} d \tau
\end{aligned}
$$

As a result, we have changed the question from looking for $\psi(t, x)$ to searching for an appropriate Green's function.

We now carry out a Fourier transformation and define

$$
\tilde{G}(x, \xi ; \omega)=\int_{-\infty}^{\infty} G(x, \xi ; t) \exp (i \omega t) d t
$$

Therefore equation (60) becomes

$$
\tilde{D} \tilde{G}(x, \xi ; \omega)=\left[-\omega^{2}-\partial_{x x}+V(x)\right] \tilde{G}(x, \xi ; \omega)=\delta(x-\xi)
$$

if the Green's function $G(x, \xi ; t)$ satisfies the conditions

$$
\lim _{t \rightarrow \infty} G(x, \xi ; t)=\lim _{t \rightarrow \infty} \partial_{t} G(x, \xi ; t)=0 .
$$

On physical grounds these assumptions are reasonable because any localized quantity is expected to be dispersed throughout the space by means of wave propagation. Mathematically, these assumptions are consistent with the Fourier transformability of the function $G(x, \xi ; t)$ which must be absolutely integrable over $\Re$ in order for $\tilde{G}(x, \xi ; \omega)$ to be well-defined. The Green's function in frequency space can be represented as

$$
\tilde{G}(x, \xi ; \omega)= \begin{cases}\frac{f(\xi ; \omega) g(x ; \omega)}{W(\omega ; g, f)} & \text { if } \xi<x \\ \frac{f(x ; \omega) g(\xi ; \omega)}{W(\omega ; g, f)} & \text { if } x<\xi\end{cases}
$$

where the function $W(\omega ; g, f)$ is the Wronskian of two linearly independent functions $f(x ; \omega)$ and $g(x ; \omega)$, that is

$$
W(\omega ; g, f)=g(x ; \omega) \partial_{x} f(x ; \omega)-f(x ; \omega) \partial_{x} g(x ; \omega)
$$


The functions $f(x ; \omega)$ and $g(x ; \omega)$ are two independent solutions of the equations

$$
\tilde{D} f(x ; \omega)=\tilde{D} g(x ; \omega)=0
$$

so that $W(\omega ; g, f)$ is independent on $x$.

Let $\tilde{\psi}(x ; \omega)$ be the Fourier transformation of the solution $\psi(t, x)$. We impose boundary conditions on the functions $f(x ; \omega)$ and $g(x ; \omega)$ so that

$$
f(x ; \omega) \propto \tilde{\psi}(x ; \omega) \quad \text { and } \quad \partial_{x} f(x ; \omega) \propto \partial_{x} \tilde{\psi}(x ; \omega)
$$

at the point $x \rightarrow-\infty$. At the other end $(x=0)$ we insist that

$$
g(x ; \omega) \propto \tilde{\psi}(x ; \omega) \quad \text { and } \quad \partial_{x} g(x ; \omega) \propto \partial_{x} \tilde{\psi}(x ; \omega) .
$$

In equation (61), after we have used the inverse Fourier transformation and interchanged the integrals with respect to $d \omega$ and $d \tau$, the representation of $\psi(t, x)$ simply becomes

$$
\psi(t, x)=\int_{-\infty}^{0}\left[G(x, \xi ; t) \partial_{t} \psi(0, \xi)+\psi(0, \xi) \partial_{t} G(x, \xi ; t)\right] d \xi .
$$

\subsection{Solution for an Approximate Potential}

We will obtain the exact solution for the scalar wave $\psi$ corresponding to the approximate potential barrier (56) in a static 3D black hole background. This entails finding the solution of the differential equation $D h(x ; \omega)=0$. This equation has two solutions [18], namely

$$
\begin{aligned}
h(x ; \omega) & =I_{\nu}(z(x)) \quad \text { or } \quad K_{\nu}(z(x)), \\
z(x) & =Z_{0} \exp (\lambda x)=\frac{\sqrt{V_{0}}}{\lambda} \exp (\lambda x),
\end{aligned}
$$

where $\nu \equiv-i \omega / \lambda$, and $I_{\nu}$ and $K_{\nu}$ are the modified Bessel functions of complex order $\nu$ [25]. At the boundary $x \rightarrow-\infty$ (the event horizon of the 3D black hole) we employ the condition

$$
f(x ; \omega)=\exp (-i \omega x) \quad x \rightarrow-\infty
$$

as usual [18]. Since this spacetime is asymptotically anti-de Sitter instead of flat, the boundary condition at spatial infinity is less trivial. Choosing Dirichlet conditions 21]

$$
g(x=0 ; \omega)=0 \quad \text { and }\left.\quad \partial_{x} g(x ; \omega)\right|_{x=0}=1,
$$

the two functions $f(x ; \omega)$ and $g(x ; \omega)$ read

$$
\begin{aligned}
& f(x ; \omega)=\mathcal{N}_{f} I_{\nu}(z(x)), \\
& g(x ; \omega)=\mathcal{N}_{g}\left[I_{\nu}\left(Z_{0}\right) K_{\nu}(z(x))-K_{\nu}\left(Z_{0}\right) I_{\nu}(z(x))\right],
\end{aligned}
$$

where the normalization coefficients $\mathcal{N}_{f}$ and $\mathcal{N}_{g}$ are given by

$$
\mathcal{N}_{f}=\left(\frac{2}{Z_{0}}\right)^{\nu} \Gamma(1+\nu) \quad \text { and } \quad \mathcal{N}_{g}=-\frac{1}{\lambda} .
$$

Now we put everything together and obtain

$$
\tilde{G}(x, \xi<x ; \omega)=\frac{I_{\nu}(z(\xi))\left[I_{\nu}\left(Z_{0}\right) K_{\nu}(z(x))-K_{\nu}\left(Z_{0}\right) I_{\nu}(z(x))\right]}{\lambda I_{\nu}\left(Z_{0}\right)} .
$$


The remainder of the problem is to bring it from the frequency domain back to the time domain.

The inverse Fourier transformation is given by the equation

$$
G(x, \xi ; t)=\frac{1}{2 \pi} \int_{-\infty}^{\infty} \tilde{G}(x, \xi ; \omega) \exp (-i \omega t) d \omega
$$

We evaluate this integral by analytically extending $\omega$ to complex values and using Cauchy's residue theorem. The contour of integration is chosen to be a large, closed semi-circle on the lower half $\omega$-plane, with center at $\omega=0$. By Jordan's lemma, the contribution from the arc of the circle goes to zero as the radius of the arc tends to infinity. The Green's function then becomes

$$
G(x, \xi ; t)=i \sum \operatorname{Res}\{\tilde{G}(x, \xi ; \omega) \exp (-i \omega t)\}
$$

where $\operatorname{Res}\{h(z)\}$ denotes the residue of the function $h(z)$ at a pole. In our case, the poles of the Green's function $\tilde{G}(x, \xi ; \omega)$ come from the term $I_{\nu}\left(Z_{0}\right)$ only. This term causes trouble because it has infinitely many zeros in the lower half $\omega$-plane. However $\nu \equiv-i \omega / \lambda=0$ is not a root of $I_{\nu}\left(Z_{0}\right)$ for positive definite $Z_{0}$ and so $G(x, \xi ; t)$ decays to zero exponentially. The dominant exponential decaying rate is determined by the zero closest to the real axis on the lower half $\omega$-plane. In general this root has a non-vanishing real part which contributes to the oscillatory nature of the falloff behaviour. As a result, the exponentially decaying Green's function $G(x, \xi ; t)$ implies that $\psi(t, x)$ also decays in this manner.

Had we chosen the Neumann condition instead of the Dirichlet condition for $g(x, \omega)$ [21], that is

$$
g(x=0 ; \omega)=1 \quad \text { and }\left.\quad \partial_{x} g(x ; \omega)\right|_{x=0}=0,
$$

the function $g(x, \omega)$ would simply become

$$
g(x ; \omega)=Z_{0}\left[I_{\nu}{ }^{\prime}\left(Z_{0}\right) K_{\nu}(z(x))-K_{\nu}{ }^{\prime}\left(Z_{0}\right) I_{\nu}(z(x))\right] .
$$

The change in $\mathcal{N}_{g}$ does not affect the result in any way because it is always cancelled in $\tilde{G}$ when the Wronskian is taken into account. The change from $I_{\nu}\left(Z_{0}\right)$ to $I_{\nu}{ }^{\prime}\left(Z_{0}\right)$ causes the singular term in $\tilde{G}$ to become $I_{\nu}{ }^{\prime}\left(Z_{0}\right)$ which also has infinitely many roots on the lower half $\omega$-plane. Again, the origin is not a root. Therefore even if we employ the Neumann condition, the solution $\psi(t, x)$ also dies out to zero at an exponential rate.

The next few graphs are the numerical results using the exponential potential. We used the same initial condition as in the numerical computation for the Schwarzschild case. Since the background is asymptotically anti-de Sitter, we integrate equation (10) numerically with the Dirichlet boundary condition at $r=\infty$ (i.e. $x=0$.) Figures 8 and 9 illustrate the falloff behaviour of the wave using different exponential potential functions. In both graphs, the initial Gaussian impulse is located at twice the black hole radius $\left(2 R_{b}\right)$ and the observation is made at $4 R_{b}$. The straight line asymptote of the ringing behaviour on the semilog graph corresponds to exponential falloff, numerically confirming that the wave exponentially decays in this asymptotically non-flat background.

A direct check of our analytic prediction against the numerical computation is problematic because no analytic procedure exists for computing the (complex) values of $\nu$ for which $I_{\nu}\left(Z_{0}\right)=0$ for fixed $Z_{0}$. Hence finding the root of $I_{-i \omega / \lambda}\left(Z_{0}\right)$ which is closest to the real axis on the lower half $\omega$-plane must be done numerically, by checking whether the root measured from the numerically generated graph (e.g. Figure 8) satisfies the equation $I_{-i \omega / \lambda}\left(Z_{0}\right)=0$ for the relevant $Z_{0}$ (e.g. $Z_{0}=3$ for Figure 8). We have checked that the numerically determined roots satisfy this criterion for various values of $Z_{0}$ for all computations carried out in the $(2+1)$-dimensional case.

Figures 10 and 11 illustrate the numerical results using the approximate potential (56) and the Neumann boundary condition at $x=0$. It is clear from the two graphs that the scalar wave also exponentially decays when the Neumann condition is used. 


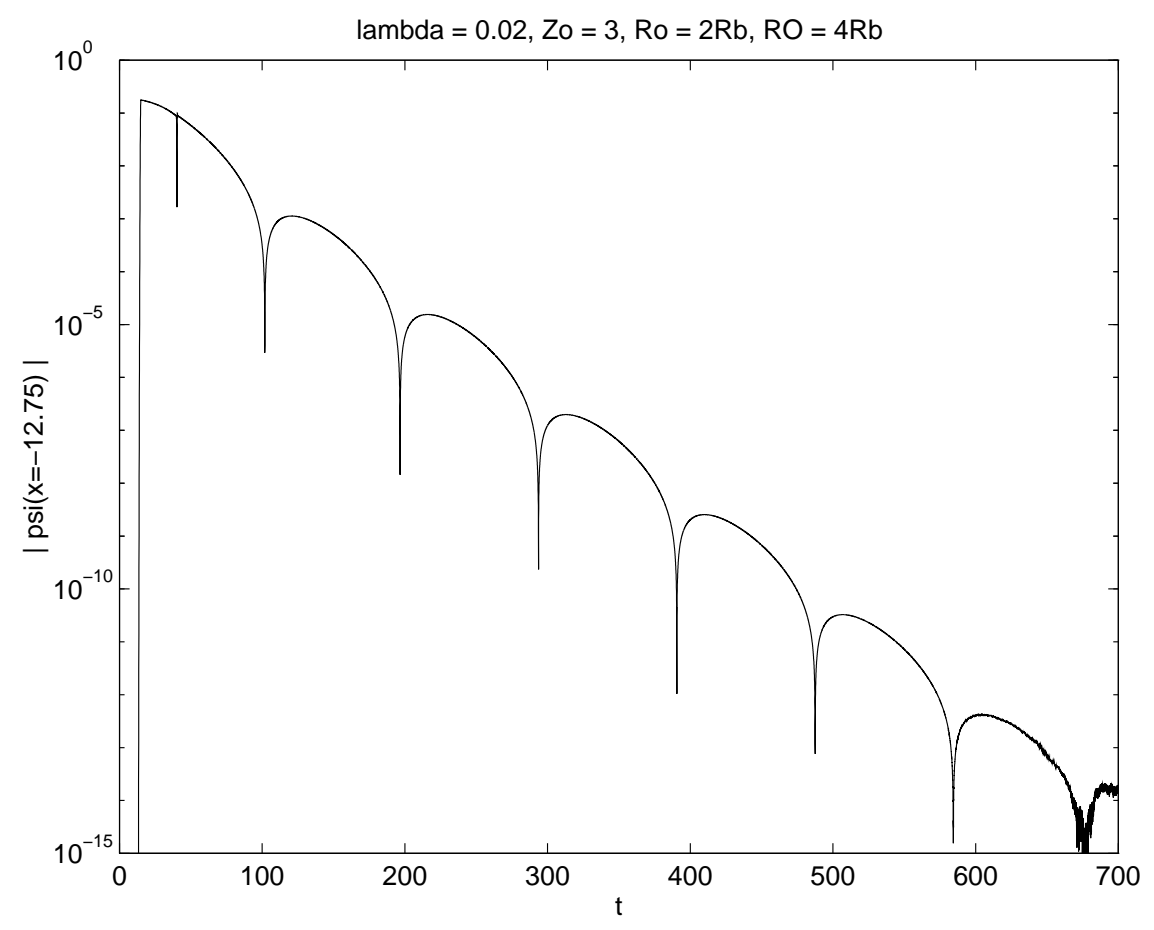

Figure 8: Exponential falloff for a conformal scalar wave using the approximate potential (56) and Dirichlet boundary condition

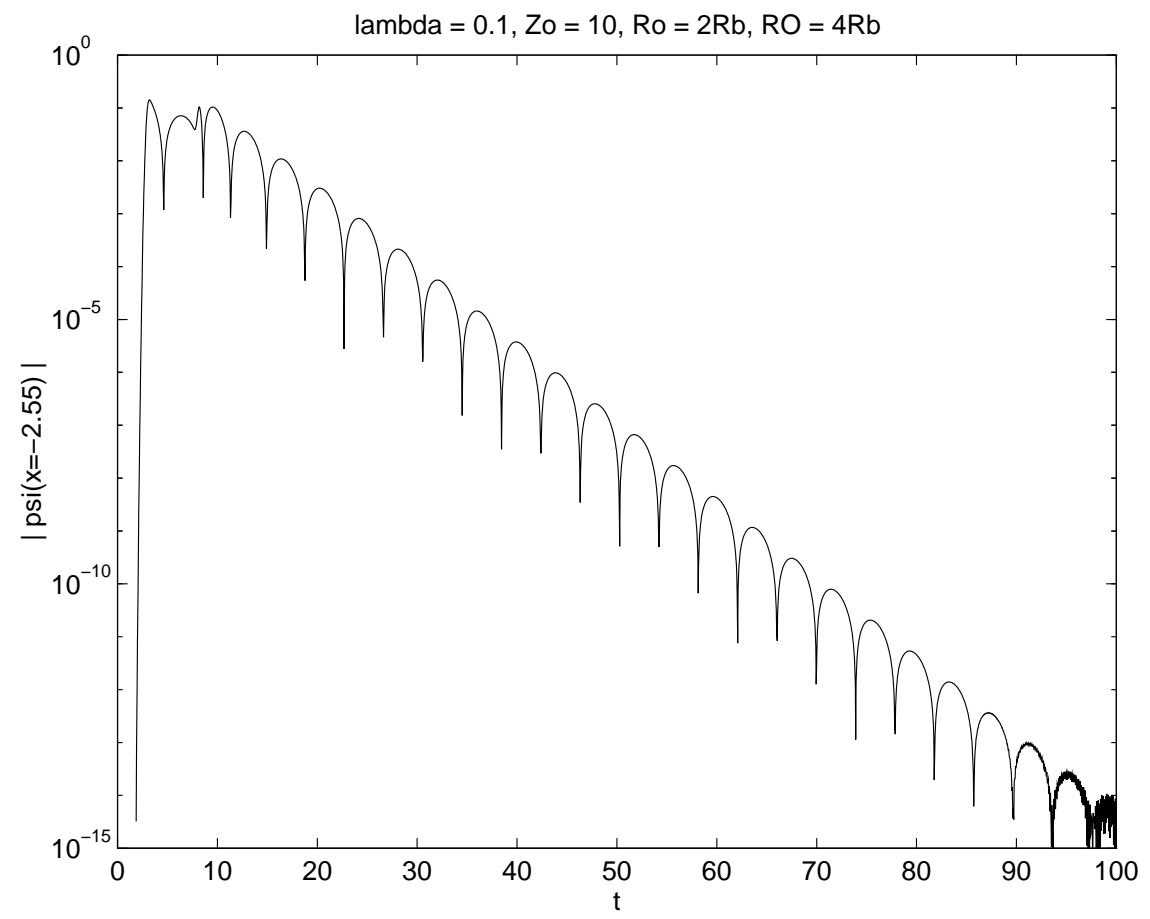

Figure 9: Another semilog graph as in figure 8 but with different parameters 


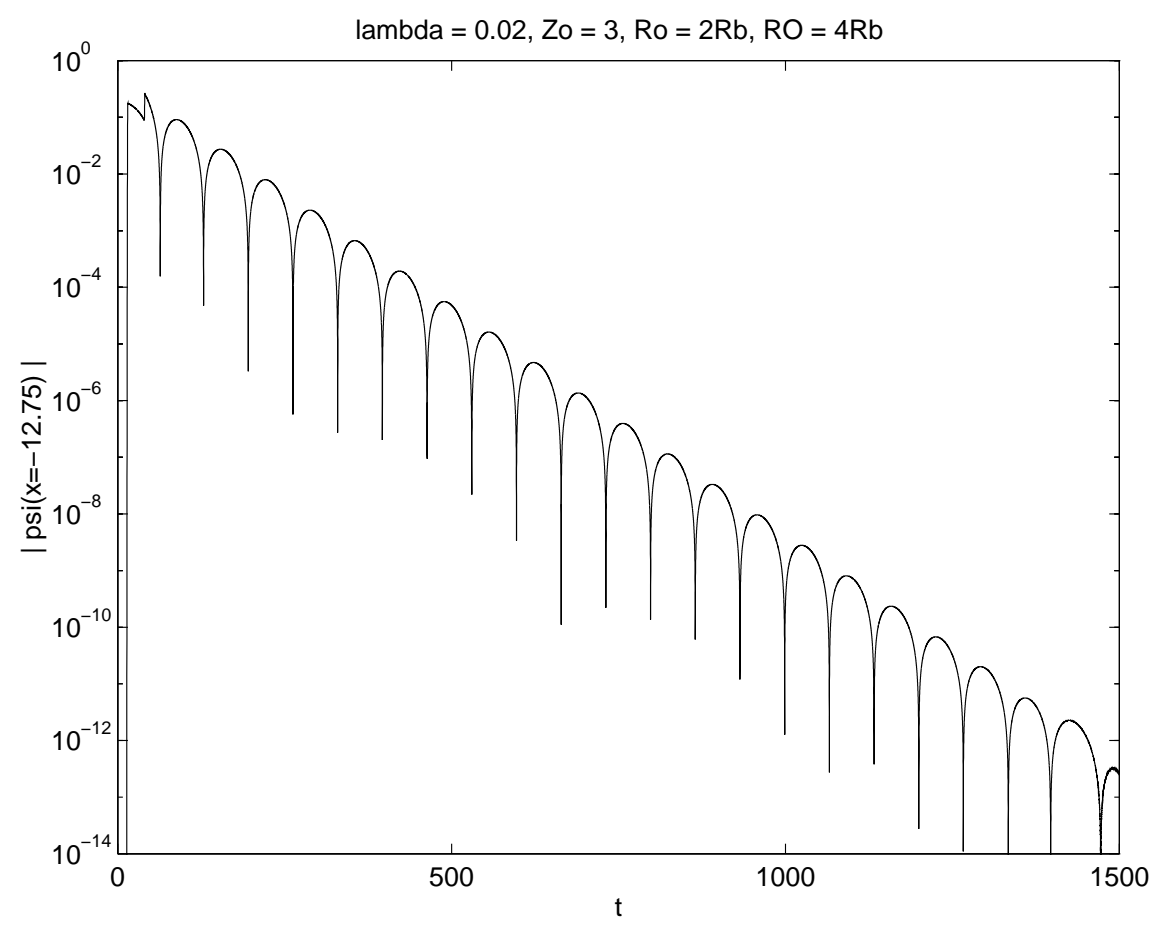

Figure 10: Exponential falloff using the Neumann condition

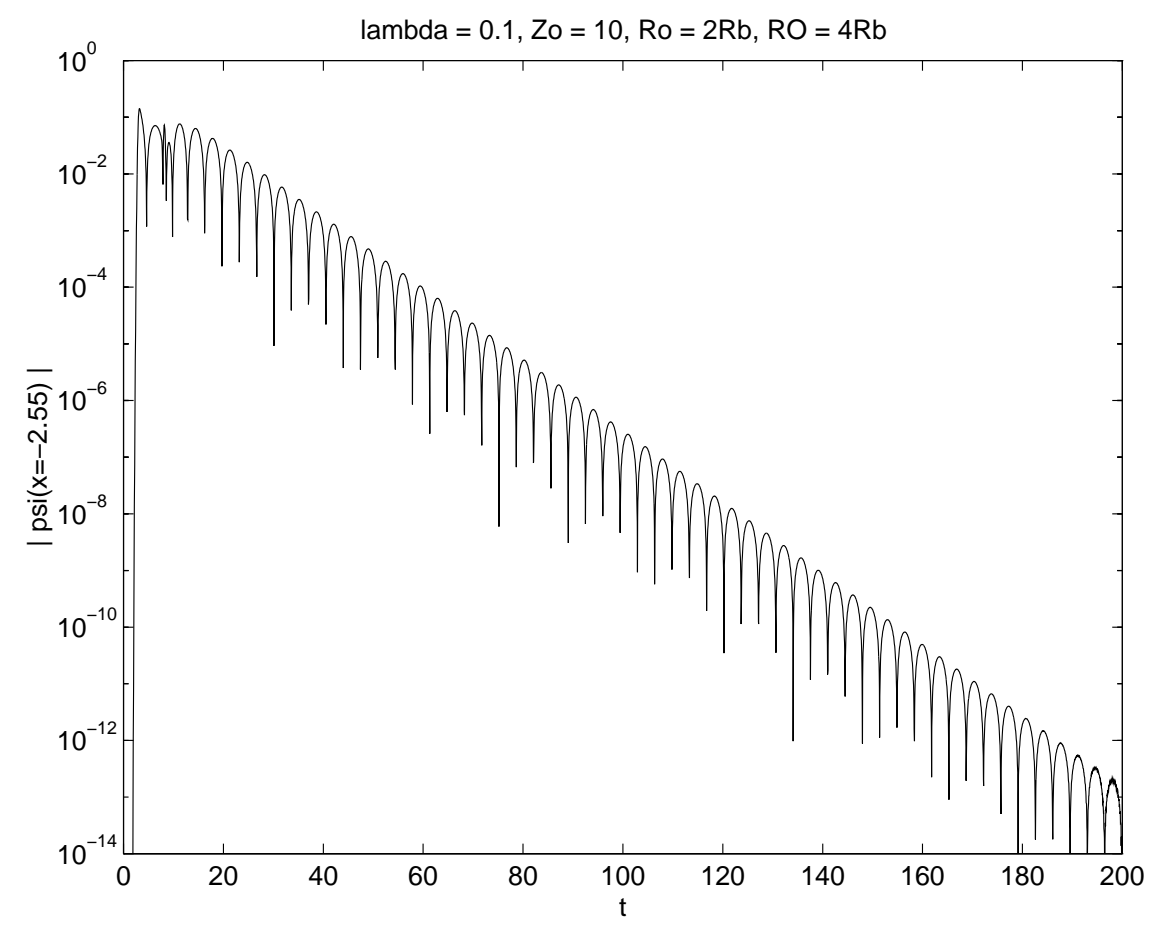

Figure 11: The same as in figure 10 but with different parameters 


\subsection{Higher order correction}

The results of the previous subsection are based upon the use of the potential barrier $V(x)=V_{0} \exp (2 \lambda x)$ which is an approximation to

$$
V(x)=V_{0} \frac{\exp (2 \lambda x)}{(1+\exp (2 \lambda x))^{2}}
$$

Since $\lambda>0$ but $x<0, \exp (2 \lambda x)$ is always less than one. Therefore $V(x)$ has a converging series expansion

$$
V(x)=V_{0}[\exp (2 \lambda x)-2 \exp (4 \lambda x)+3 \exp (6 \lambda x)-4 \exp (8 \lambda x)+O(\exp (10 \lambda x))]
$$

As a result we may iteratively solve the wave equation, treating $V_{0} \exp (2 \lambda x)$ as the lowest order term, - $2 V_{0} \exp (4 \lambda x)$ as the first correction to this approximation, and so on.

For clarity, we define

$$
\begin{aligned}
V_{0}(x) & \equiv V_{0} \exp (2 \lambda x) \\
V_{1}(x) & \equiv-2 V_{0} \exp (4 \lambda x) \\
\tilde{D} & \equiv-\omega^{2}-\partial_{x x}+V_{0}(x)+V_{1}(x) \\
\tilde{D}_{0} & \equiv-\omega^{2}-\partial_{x x}+V_{0}(x)
\end{aligned}
$$

Therefore the equation $\tilde{D} f(x ; \omega)=0$ has a representation

$$
f(x ; \omega)=f_{0}(x ; \omega)+\int_{-\infty}^{x} \frac{U_{+}(x) U_{-}(\xi)-U_{-}(x) U_{+}(\xi)}{W\left(\omega ; U_{-}, U_{+}\right)} V_{1}(\xi) f(\xi ; \omega) d \xi
$$

at $x=-\infty$. The functions $U_{+}(x)$ and $U_{-}(x)$ satisfy the equation $\tilde{D}_{0} U_{+}(x)=\tilde{D}_{0} U_{-}(x)=0$. The other function $f_{0}(x ; \omega)$ satisfies the same equation as $U_{+}(x)$ and $U_{-}(x)$ and also satisfies the boundary conditions at $x \rightarrow-\infty$. In other words, $f_{0}(x ; \omega)$ is the solution (75) in our case. Similarly the solution around $x=0$ is

$$
g(x ; \omega)=g_{0}(x ; \omega)-\int_{x}^{0} \frac{U_{+}(x) U_{-}(\xi)-U_{-}(x) U_{+}(\xi)}{W\left(\omega ; U_{-}, U_{+}\right)} V_{1}(\xi) g(\xi ; \omega) d \xi,
$$

where $g_{0}(x ; \omega)$ is just (76). We first compute the Wronskian $W(\omega ; g, f)$ for $f(x ; \omega)$ and $g(x ; \omega)$ above. Since this Wronskian is $x$-independent, the simplest way to compute it is to evaluate the quantity at the point $x=0$. It is not difficult to show that

$$
W(\omega ; g, f)=W\left(\omega ; g_{0}, f_{0}\right)+\left.W\left(\omega ; g_{0}, f\right)(x)\right|_{x=-\infty} ^{x=0}=W\left(\omega ; g_{0}, f_{0}\right)-\left.W\left(\omega ; g, f_{0}\right)(x)\right|_{x=-\infty} ^{x=0} .
$$

Since $g(x ; \omega)$ and $f(x ; \omega)$ satisfy a differential equation which differs from that satisfied by $g_{0}(x ; \omega)$ and $f_{0}(x ; \omega)$, the Wronskians $W\left(\omega ; g_{0}, f\right)$ and $W\left(\omega ; g, f_{0}\right)$ are functions of $x$ in general. Therefore the correction $V_{1}(x)$ in the potential barrier induces an extra ( $x$-independent) term in the Wronskian $W(\omega ; g, f)$.

The first Born approximations for $f(x ; \omega)$ and $g(x ; \omega)$ read

$$
f(x ; \omega) \approx f_{1}(x ; \omega)=f_{0}(x ; \omega)+\frac{2 \lambda^{2}}{V_{0}} \int_{0}^{z(x)}\left[K_{\nu}(z(x)) I_{\nu}(s)-I_{\nu}(z(x)) K_{\nu}(s)\right] \hat{f}_{0}(s ; \omega) s^{3} d s
$$

and

$$
g(x ; \omega) \approx g_{1}(x ; \omega)=g_{0}(x ; \omega)-\frac{2 \lambda^{2}}{V_{0}} \int_{z(x)}^{Z_{0}}\left[K_{\nu}(z(x)) I_{\nu}(s)-I_{\nu}(z(x)) K_{\nu}(s)\right] \hat{g_{0}}(s ; \omega) s^{3} d s
$$


if we choose $U_{-}(x)=I_{\nu}(z(x))$ and $U_{+}(x)=K_{\nu}(z(x))$. The functions $\hat{f}_{0}(s ; \omega)$ and $\hat{g}_{0}(s ; \omega)$ are defined via the equations

$$
\begin{aligned}
& \hat{f}_{0}(z(x) ; \omega)=f_{0}(x ; \omega)=\mathcal{N}_{f} I_{\nu}(z(x)) \\
& \hat{g}_{0}(z(x) ; \omega)=g_{0}(x ; \omega)=\mathcal{N}_{g}\left[I_{\nu}\left(Z_{0}\right) K_{\nu}(z(x))-K_{\nu}\left(Z_{0}\right) I_{\nu}(z(x))\right]
\end{aligned}
$$

As a result, the Wronskian $W(\omega ; g, f)$ can be approximated by

$$
W(\omega ; g, f) \approx W\left(\omega ; g_{0}, f_{0}\right)+\left.W\left(\omega ; g_{0}, f_{1}\right)(x)\right|_{-\infty} ^{0}=W\left(\omega ; g_{0}, f_{0}\right)+\int_{-\infty}^{0} g_{0}(x ; \omega) f_{0}(x ; \omega) V_{1}(x) d x
$$

This Wronskian has roots on the lower half $\omega$-plane. Thus there is quasi-normal ringing effect in the wave tail in this $3 \mathrm{D}$ black hole configuration with the approximate potential function $V(x) \approx V_{0}(x)+V_{1}(x)$. Moreover, since $g_{1}$ and $f_{1}$ have no cut, the falloff rate of the conformal wave is also exponential.

The shape of the exact potential function $V(x)$ is given on figure 12 with parameters $\lambda=0.02$ and $Z_{0}=3$. Figures 13 and 14 are results of the numerical calculations using the exact 3D black hole potential (56). The methodology underlying these computations is identical to the previous case that uses the approximate exponential potential function. The parameters and boundary conditions used in this case are also the same as the cases in figures 8 and 9 . Once again the numerical calculations show the exponential falloff of the scalar wave in the static 3D background.

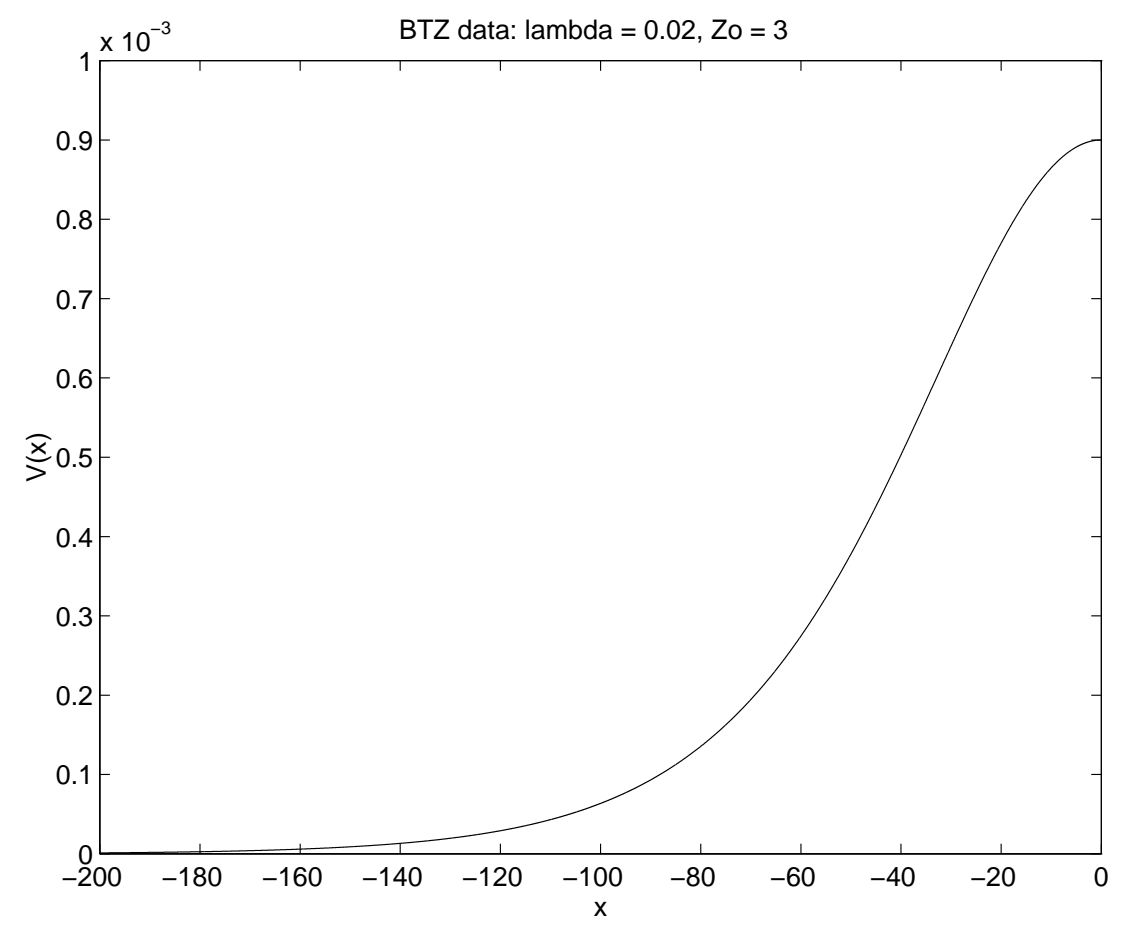

Figure 12: The shape of the exact 3D black hole potential $V(x)$

\subsection{Spinning 3D Black Hole Background}

When $J \neq 0$, i.e. the black hole rotates, we also find a late time exponential decay rate, as we shall now demonstrate. 


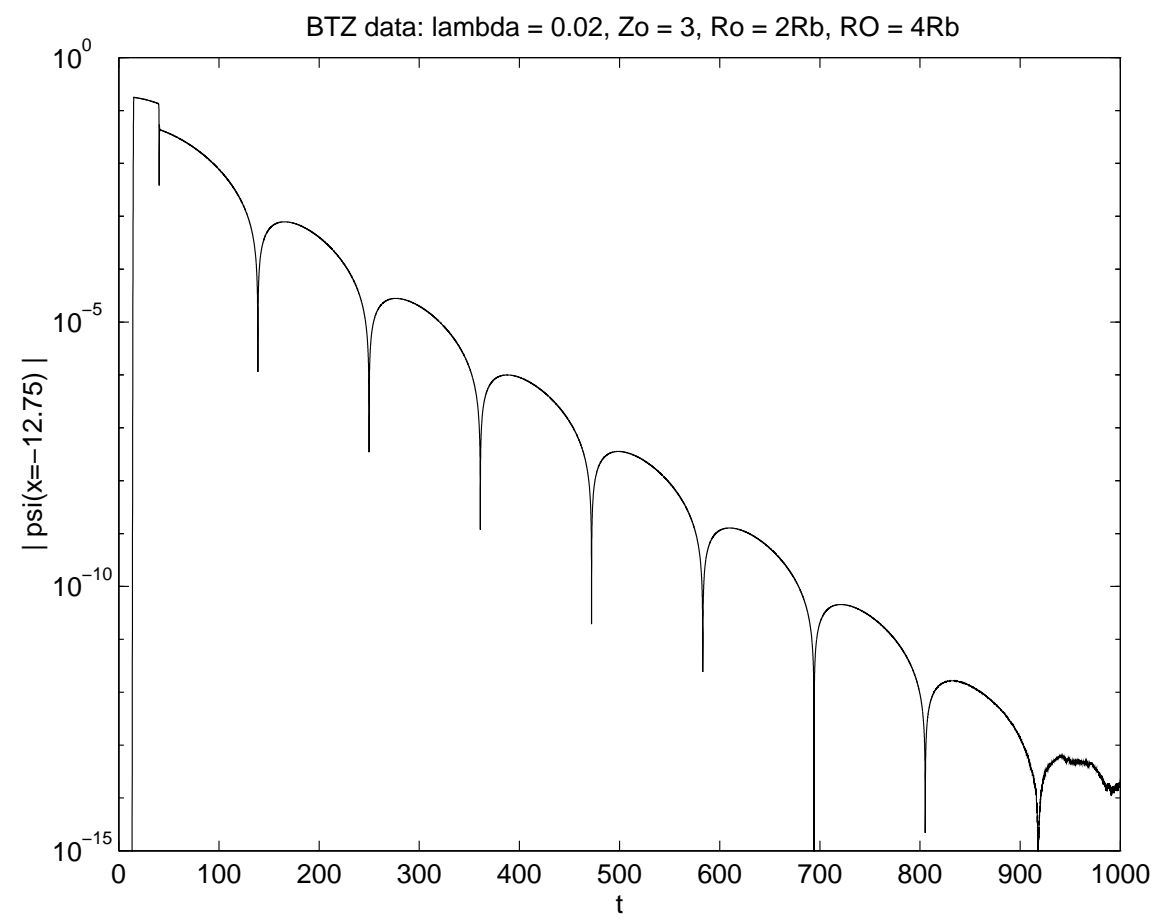

Figure 13: Exponential decay of the scalar wave $\psi$ in the static 3D black hole background

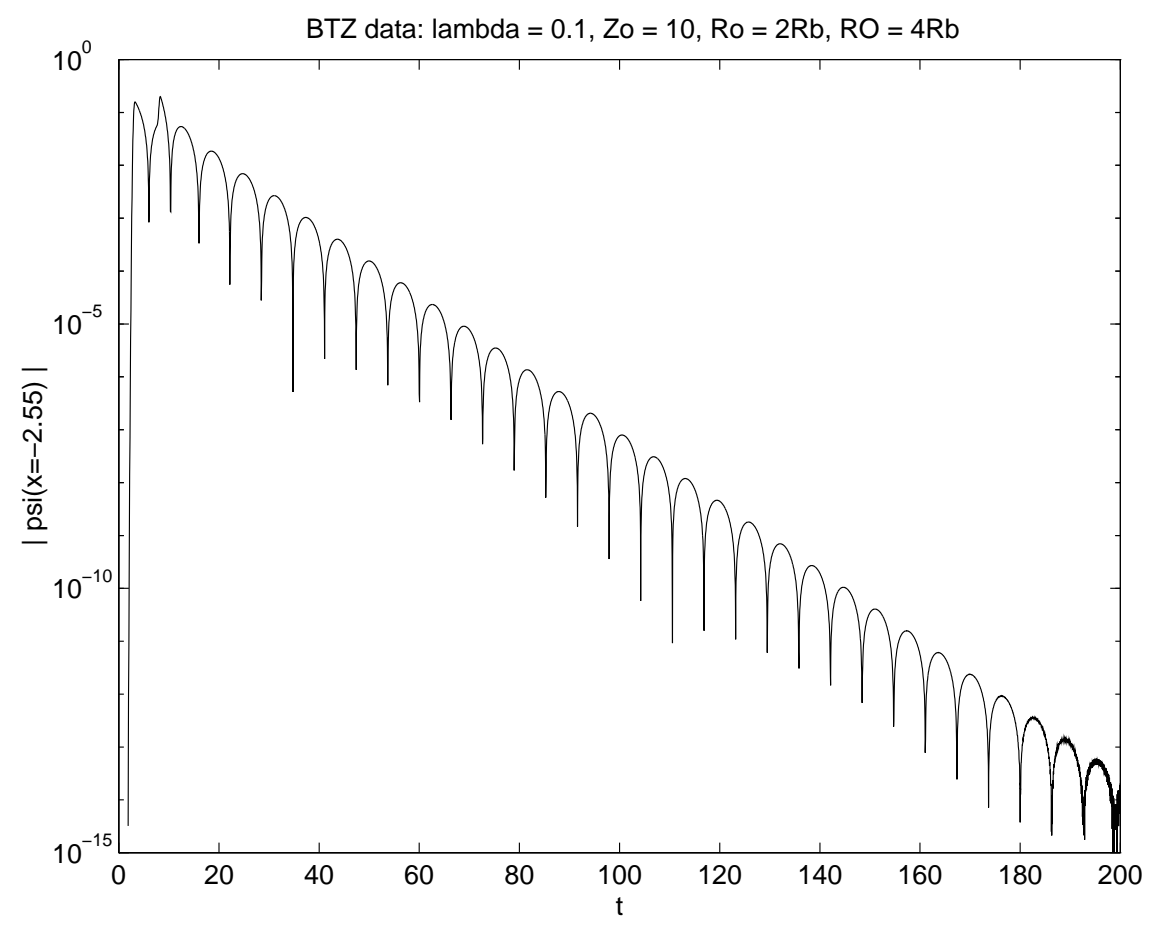

Figure 14: Scalar wave decay as in fig 13 but with different parameters 
If we assume that

$$
\Psi(t, r, \phi)=\frac{\psi(t, r)}{\sqrt{r}}
$$

that is to say there is no "spherical harmonic" component to the wave, the conformal scalar wave equation $\nabla^{2} \Psi=R \Psi / 8$ will reduce to (10). The tortoise coordinate $x$ is defined as before but the potential barrier becomes

$$
V_{e}(x(r))=-\frac{3|\Lambda|}{4}+\frac{1}{2 r} \partial_{r} N(r)-\frac{N(r)}{4 r^{2}} .
$$

In this spinning case, the black hole has two horizons $R_{ \pm}$which are given by the equation

$$
R_{ \pm}^{2}=\frac{1}{2|\Lambda|}\left[M \pm \sqrt{M^{2}-|\Lambda| J^{2}}\right]
$$

The lapse function $N(r)$ can then be written in terms of $R_{+}$and $R_{-}$as

$$
N(r)=\frac{|\Lambda|}{r^{2}}\left(r^{2}-R_{+}^{2}\right)\left(r^{2}-R_{-}^{2}\right) .
$$

As a result, the tortoise coordinate reads

$$
x(r)=\frac{1}{2|\Lambda|\left(R_{+}{ }^{2}-R_{-}{ }^{2}\right)}\left[R_{+} \ln \left(\frac{r-R_{+}}{r+R_{+}}\right)-R_{-} \ln \left(\frac{r-R_{-}}{r+R_{-}}\right)\right]
$$

which has an inverse of the form

$$
\begin{aligned}
\frac{R_{+}}{r(x)} & =\frac{Y}{1-\sigma^{2}} \sum_{n=0}^{\infty} a_{n}(\sigma) Y^{2 n} \\
\sigma & =\frac{R_{-}}{R_{+}}<1 \\
Y & =\frac{1-\exp (2 \lambda x)}{1+\exp (2 \lambda x)}<1, \\
\lambda & =|\Lambda| R_{+}\left(1-\sigma^{2}\right)>0 .
\end{aligned}
$$

The coefficients $a_{n}(\sigma)$ which read

$$
\begin{aligned}
a_{0}(\sigma)=1, & a_{1}(\sigma) & =-\sigma^{2} \frac{3-\sigma^{2}}{3\left(1-\sigma^{2}\right)^{2}}, \\
a_{2}(\sigma)=\sigma^{4} \frac{25-17 \sigma^{2}+3 \sigma^{4}}{15\left(1-\sigma^{2}\right)^{4}}, & a_{3}(\sigma) & =-\sigma^{6} \frac{1008-1039 \sigma^{2}+368 \sigma^{4}-45 \sigma^{6}}{315\left(1-\sigma^{2}\right)^{6}},
\end{aligned}
$$

are of order $O\left(\sigma^{2 n}\right)$. If $\sigma$ is small enough, we can employ the approximation

$$
\frac{1}{r(x)} \approx \frac{Y}{R_{+}}
$$

If we substitute this approximation into the potential barrier $V(x)$, we will obtain

$$
\begin{aligned}
V(x) \approx & \Lambda^{2} \frac{\left({R_{+}}^{2}-R_{-}{ }^{2}\right)\left({R_{+}}^{2}-4 R_{-}{ }^{2}\right)}{R_{+}{ }^{2}} \frac{\exp (2 \lambda x)}{(1+\exp (2 \lambda x))^{2}} \\
& +12 \Lambda^{2}\left(2{R_{+}}^{2}-3 R_{-}{ }^{2}\right) \frac{R_{-}{ }^{2}}{{R_{+}}^{2}} \frac{\exp (4 \lambda x)}{(1+\exp (2 \lambda x))^{4}}+80 \Lambda^{2} \frac{R_{-}{ }^{4}}{R_{+}{ }^{2}} \frac{\exp (6 \lambda x)}{(1+\exp (2 \lambda x))^{6}} .
\end{aligned}
$$




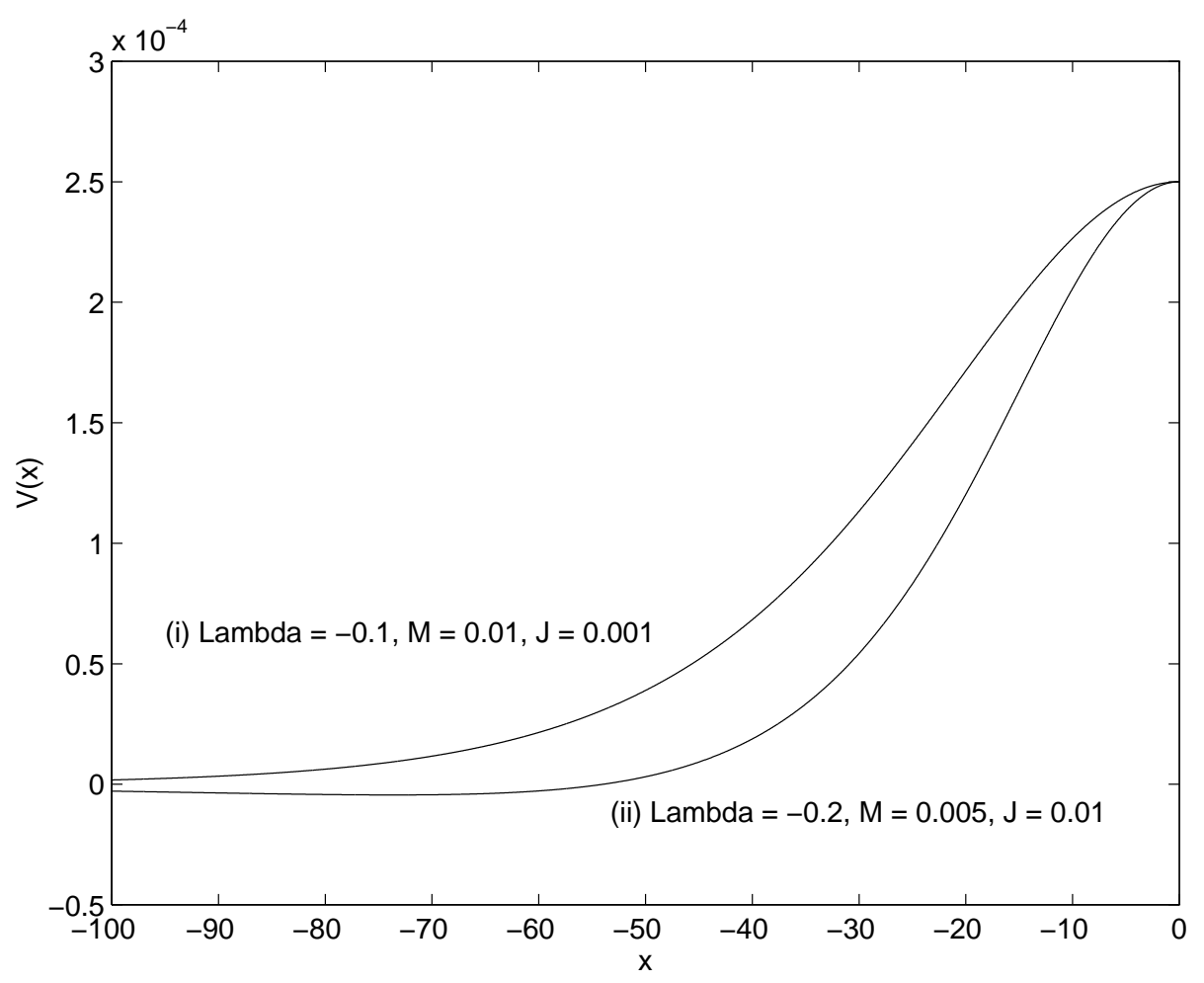

Figure 15: Potential function $V(x)$ of two spinning 3D black hole backgrounds with (i) $|\Lambda| J^{2} / M^{2}=10^{-3}$ and (ii) $|\Lambda| J^{2} / M^{2}=0.8$

It is obvious that the first approximation of this potential has a form $V(x) \approx V_{0} \exp (2 \lambda x)$ while the second one is $V(x) \approx V_{0} \exp (2 \lambda x)+V_{1} \exp (4 \lambda x)$. According to our previous analysis, we may conclude that the decay rate at late time is also exponential rather than a power-law.

Figure 15 is the graph of the potential functions of this spinning case with zero angular harmonic. One can show that when the ratio $|\Lambda| J^{2} / M^{2}>16 / 25, V_{e}$ becomes negative for some $r>R_{+}$. This behaviour is illustrated in figure 15, where $V(x)$ becomes negative when $|x|$ is sufficiently large. In figure 16, we can see that the potential functions in both cases vanish at an exponential rate towards the event horizon. As usual, we put a Gaussian impulse at a distance twice of the outer horizon $R_{+}$and the numerical response of the scalar waves over time at a distance $4 R_{+}$is shown in figure 17. This figure shows an exponential decay of the wave although its appearance differs from the previous graphs because there are no angular harmonics (i.e. $l=0$ ). Indeed if we set $l=0$ in the static 3D black hole case, we find that the falloff also looks like that in figure 17 because the pole that is closest to the real $\omega$-axis for the Green's function in frequency domain has no real part.

\section{Schwarzschild-Anti-de Sitter Background}

Since the 3D black hole spacetime is asymptotically anti-de Sitter, one might expect that the late time falloff behaviour of any scalar wave in Schwarzschild-anti-de Sitter (SAdS) background has similar behaviour. In fact, the situation is quite different from the $3 \mathrm{D}$ case, in part because of the different dimensionality and in part because it is not possible to solve the wave equation (10) exactly in this background. Although the potential function $V_{e}(r)$ in this case reads

$$
V_{e}(r)=\frac{2|\Lambda|}{3}(1-6 \xi)+\frac{l(l+1)}{r^{2}}+\frac{2 M}{r^{3}}
$$




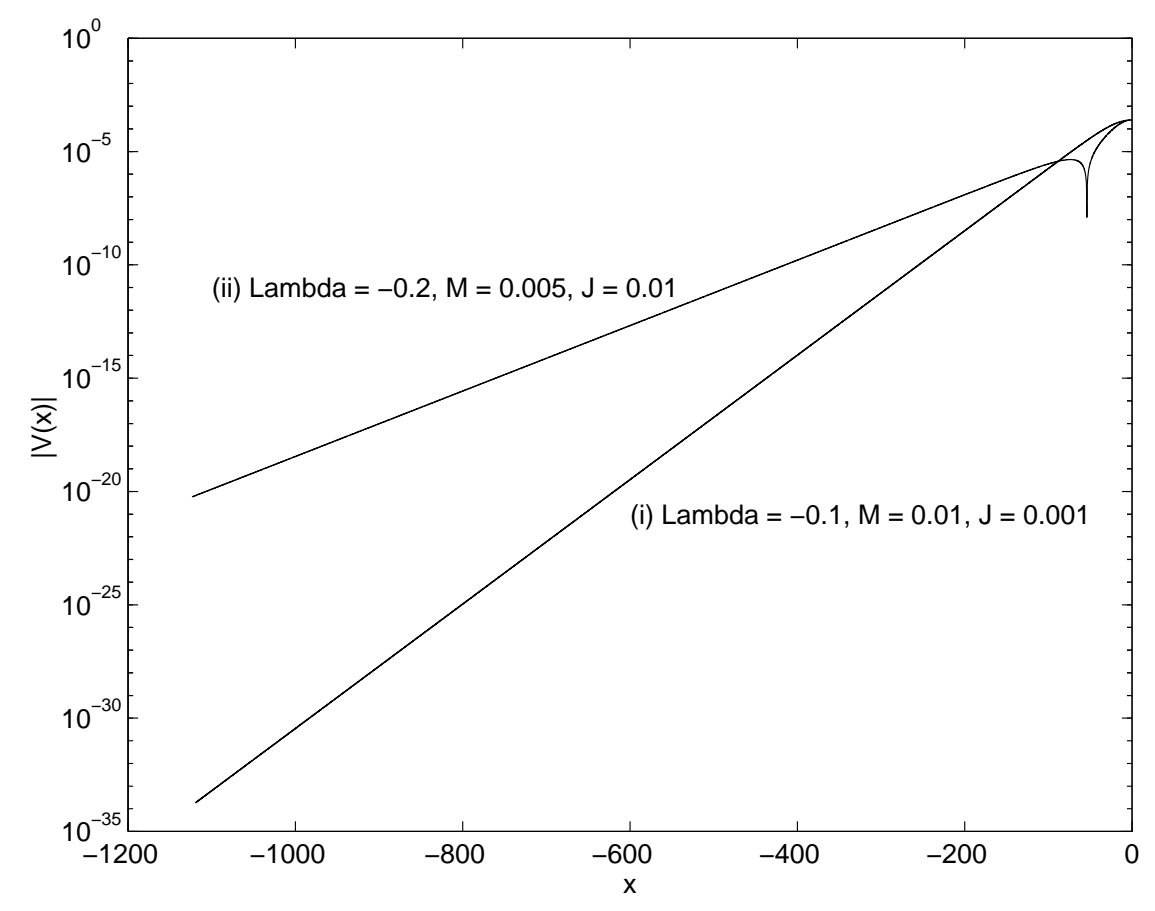

Figure 16: Decaying exponential behaviour of $|V(x)|$ near the black hole event horizon

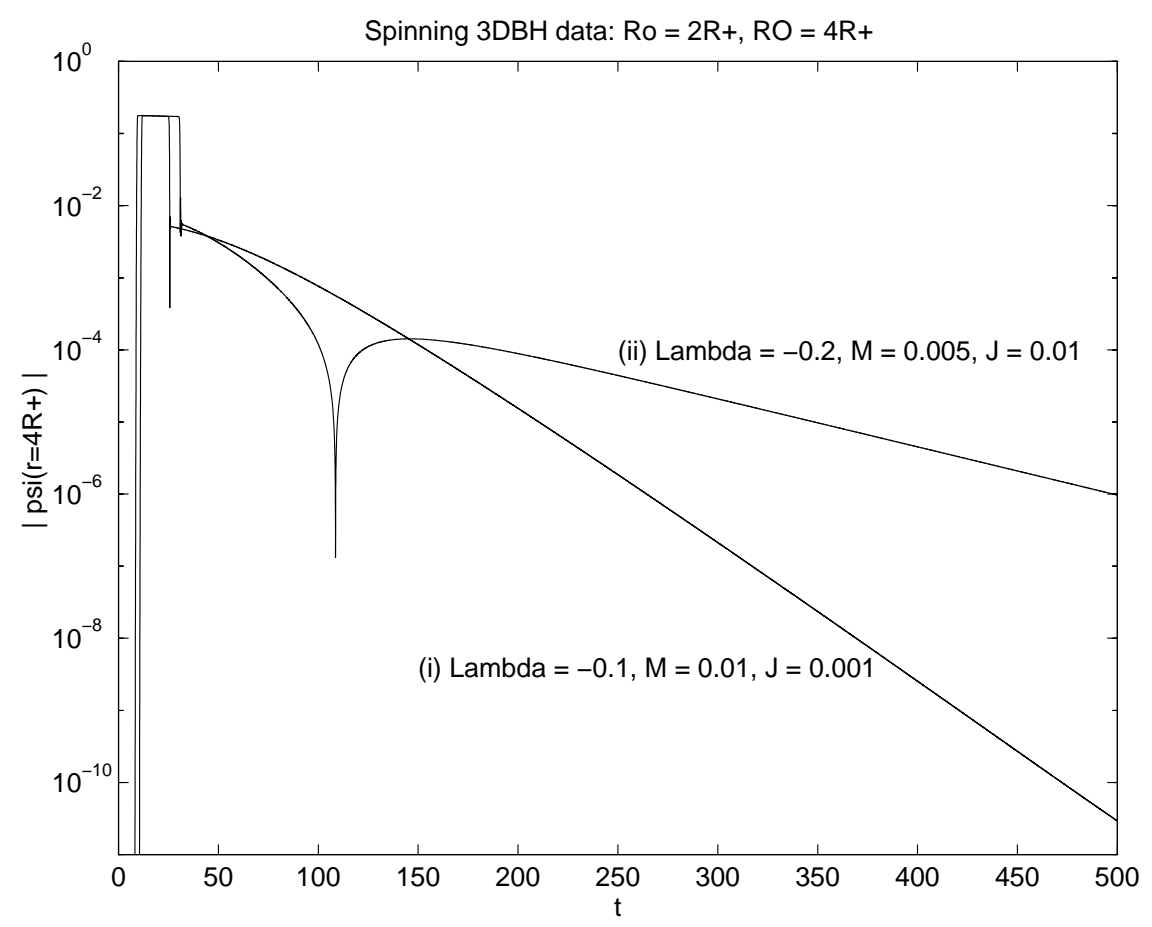

Figure 17: Exponential decay of conformal scalar waves in spinning 3D black hole geometry 
which is quite similar to (53) in the 3D case, we cannot write $r$ as a function of $x$ in a closed form. That is to say given the lapse function

$$
N(r)=\frac{|\Lambda|}{3} r^{2}+1-\frac{2 M}{r}=\frac{|\Lambda|}{3} r^{2}+1-\frac{R_{b}\left(3+|\Lambda| R_{b}^{2}\right)}{3 r},
$$

where $\Lambda<0$ and the black hole radius $R_{b}$ satisfies the equation $N\left(R_{b}\right)=0$, the tortoise coordinate

$$
\begin{aligned}
x= & \frac{R_{b}}{2\left(1+|\Lambda| R_{b}{ }^{2}\right)} \ln \left|\frac{\left(r-R_{b}\right)^{2}}{r^{2}+R_{b} r+R_{b}{ }^{2}+3 /|\Lambda|}\right| \\
& +\frac{\sqrt{3}\left(2+|\Lambda| R_{b}{ }^{2}\right)}{\sqrt{|\Lambda|}\left(1+|\Lambda| R_{b}{ }^{2}\right) \sqrt{4+|\Lambda| R_{b}{ }^{2}}}\left[\arctan \left(\frac{\sqrt{|\Lambda|}\left(2 r+R_{b}\right)}{\sqrt{3} \sqrt{4+|\Lambda| R_{b}{ }^{2}}}\right)-\frac{\pi}{2}\right]
\end{aligned}
$$

has no closed form inverse, and so we are unable to write down an explicit expression for the potential $V(x)$.

For the remainder of this section we set $\xi=1 / 6$ (i.e. we consider a conformal scalar field propagating on an SAdS background).

The most important difference between the 3D black hole background and SAdS background is the shape of the potential function $V(x)$. In both cases the potential functions are decreasing in an exponential manner toward the horizon. In the 3D case (either spinning or static) this function attains a maximum at a distance $r=\infty(x=0)$. When the background is SAdS, this is no longer true because spatial infinity (which is still given by $x=0$ ) is not the place at which $V(x)$ has an absolute maximum (for $x \in \Re^{-}$). As with the Schwarzschild black hole (figure 2), the potential function $V(x)$ attains a maximum not far away from the event horizon. In the SAdS case, the shape of the potential function $V(x)$ is given in figure 18. Unlike the Schwarzschild case, the tortoise coordinate $x$ for the SAdS background is bounded above. Eventually all the outgoing waves that leave the black hole region will return towards it due to the boundary condition at $x=0$. The returning wave will then reflect off of the potential barrier back toward spatial infinity. This is completely different behaviour from the $3 \mathrm{D}$ case, in which the incoming wave from spatial infinity $x=0$ continues its journey to the black hole unhindered.

We can see from figure 18 that when $|\Lambda|$ is small (barriers (a)-(c)), the barrier maximum moves to the left, lengthening the travelling time from this maximum to spatial infinity. When $l$ vanishes (case $(c)$ and $(\mathrm{C})$ ), $V(0)=0$ because $V(0)=l(l+1)|\Lambda| / 3$ in general. Therefore barriers (a) and (b) have $V(0) \neq 0$ although this feature is not apparent on the graph due to the small size of $|\Lambda|$. The barrier height is considerably higher than the magnitude of $V(x)$ at $x=0$, and this feature becomes more pronounced for large $l$. This causes the scalar wave to bounce back and forth in the region outside the barrier. However part of the scalar wave can surmount the barrier (thereby going into the black hole) because the barrier height is still finite. However it takes a long time for a significant portion of the wave to enter the black hole.

We solve the wave equation in this SAdS background numerically. The results of the numerical integration of equation (10) using (110) and (111) are given in the next few graphs. Figures 19 and 20 show the falloff behaviour of a conformal scalar wave with $l=0$ initially located at a distance $r=2 R_{b}$. The computation for figure 19 uses the Dirichlet condition at $x=0$ but Neumann boundary condition is employed for figure 20. Since the cosmological constant $|\Lambda|$ was chosen to be relatively small in both cases, namely $|\Lambda|=10^{-4}$, we can see on both graphs that there is clearly an inverse power-decay behaviour. According to the graph, this power-decay rate is roughly $t^{-3}$ which agrees with the one in Schwarzschild case. However this inverse power-decay does not last very long after the return of the outgoing wave from spatial infinity. Both diagrams show this returning wavefront.

For small $|\Lambda|$ and nonzero $l$, the falloff behaviour resembles the case of $l=0$. Initially there is a ringing effect (due to the quasi-normal modes) followed by inverse power-decay behaviour as shown in figures 21 and 22 . 


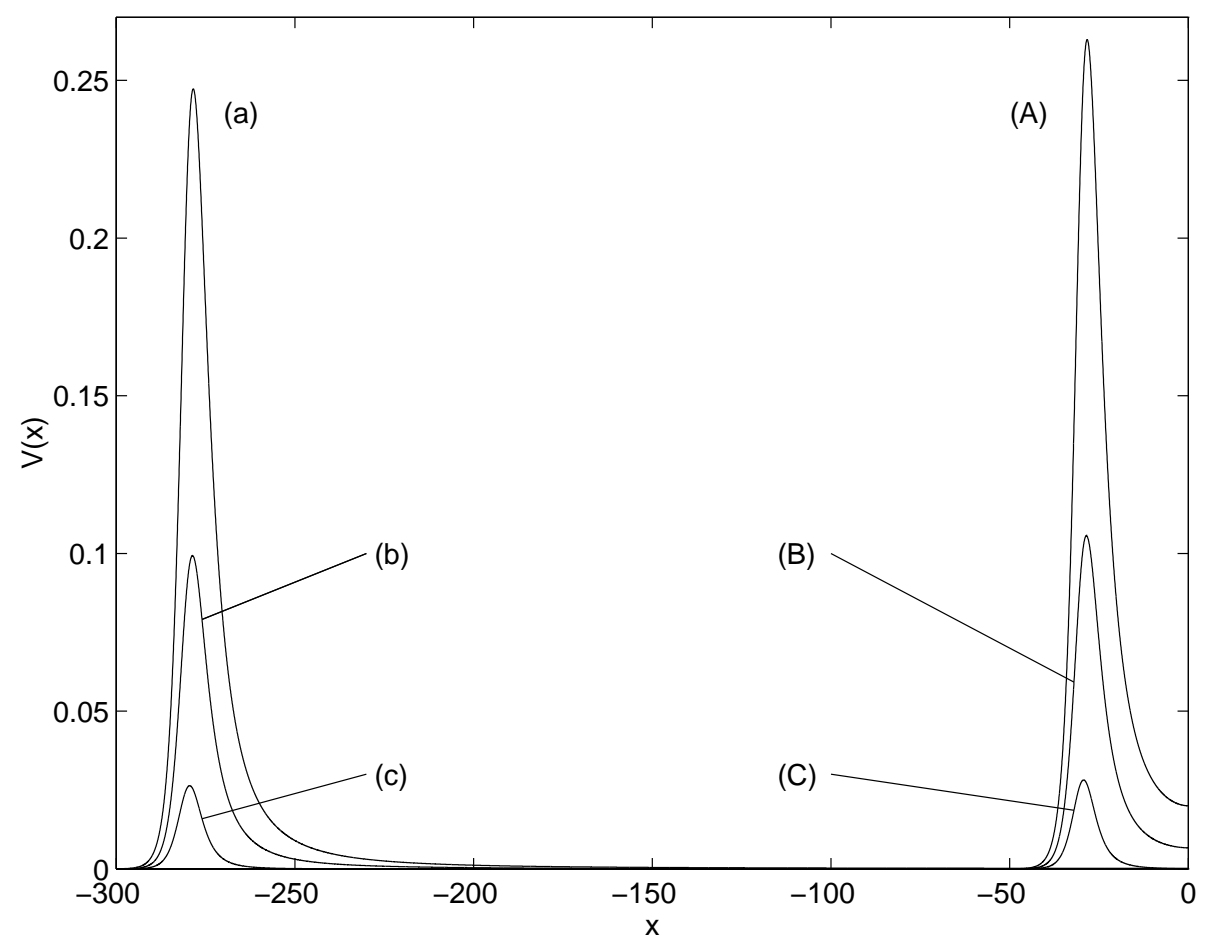

Figure 18: Potential functions $V(x)$ for the SAdS background. The six potentials are generated with the parameters (a) $\Lambda=-10^{-4}, l=2$, (b) $\Lambda=-10^{-4}, l=1$, (c) $\Lambda=-10^{-4}, l=0$, (A) $\Lambda=-10^{-2}, l=2$, (B) $\Lambda=-10^{-2}, l=1,(\mathrm{C}) \Lambda=-10^{-2}, l=0$.

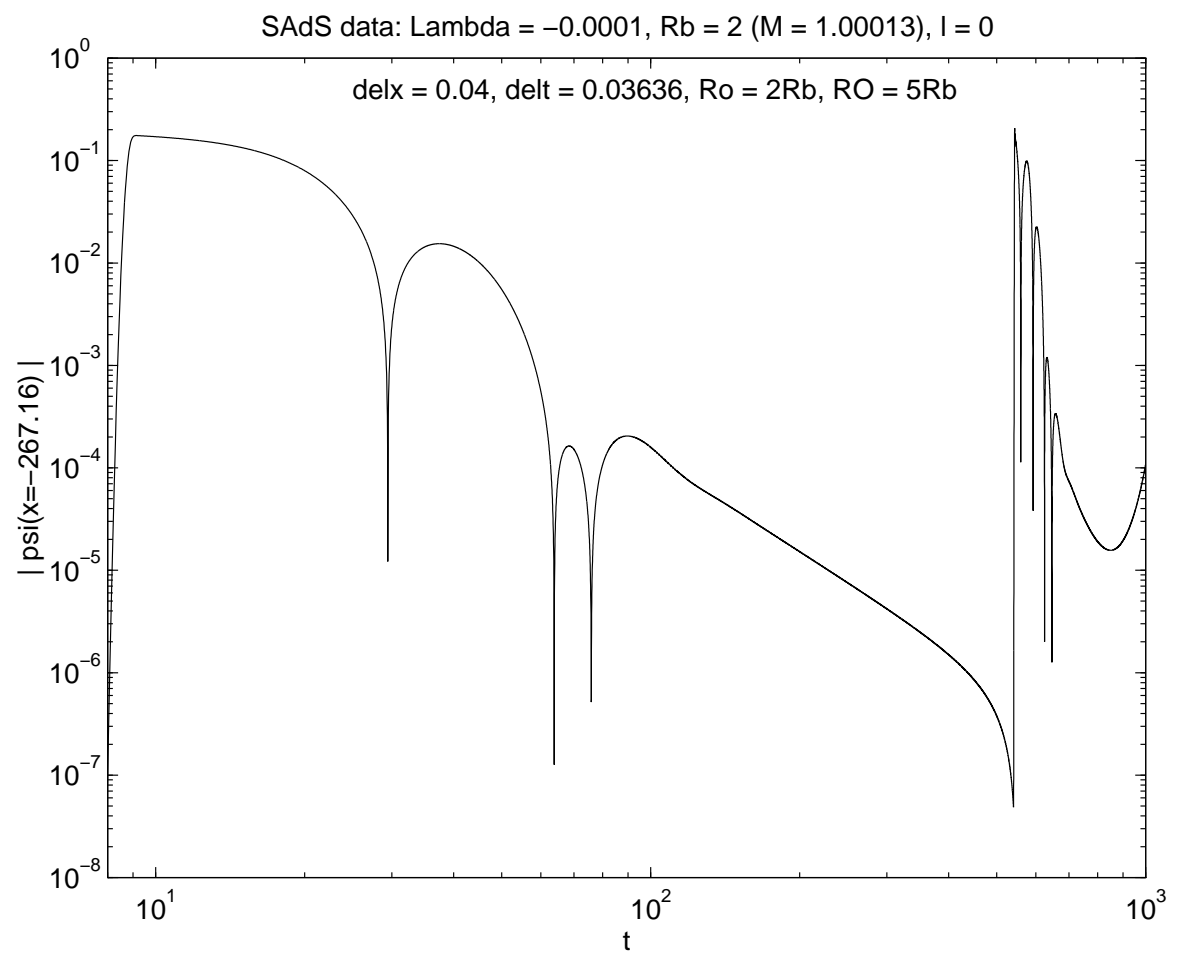

Figure 19: Scalar wave of $l=0$ decays away in SAdS background using Dirichlet condition at $x=0$ 


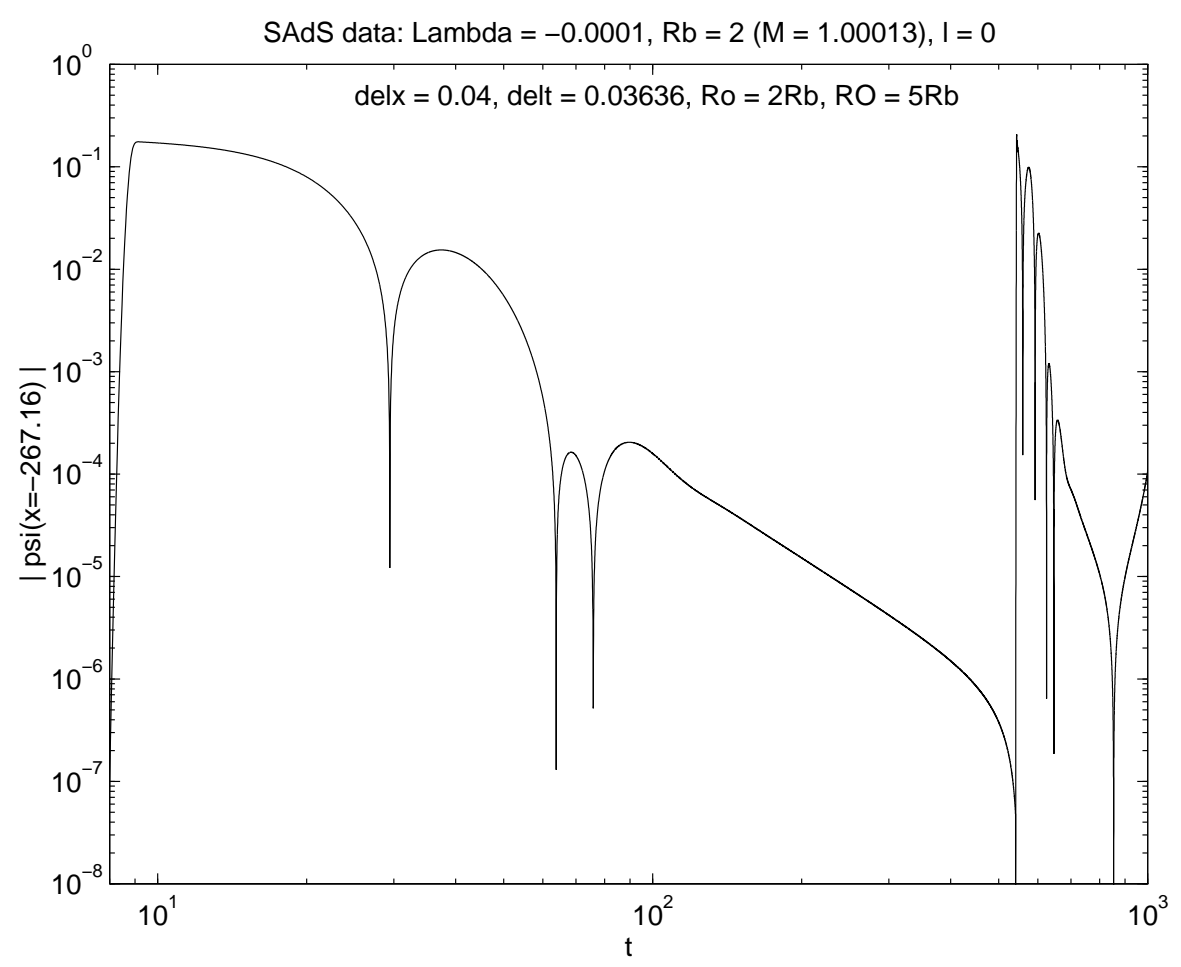

Figure 20: Scalar wave falloff pattern of $l=0$ using Neumann condition spatial infinity

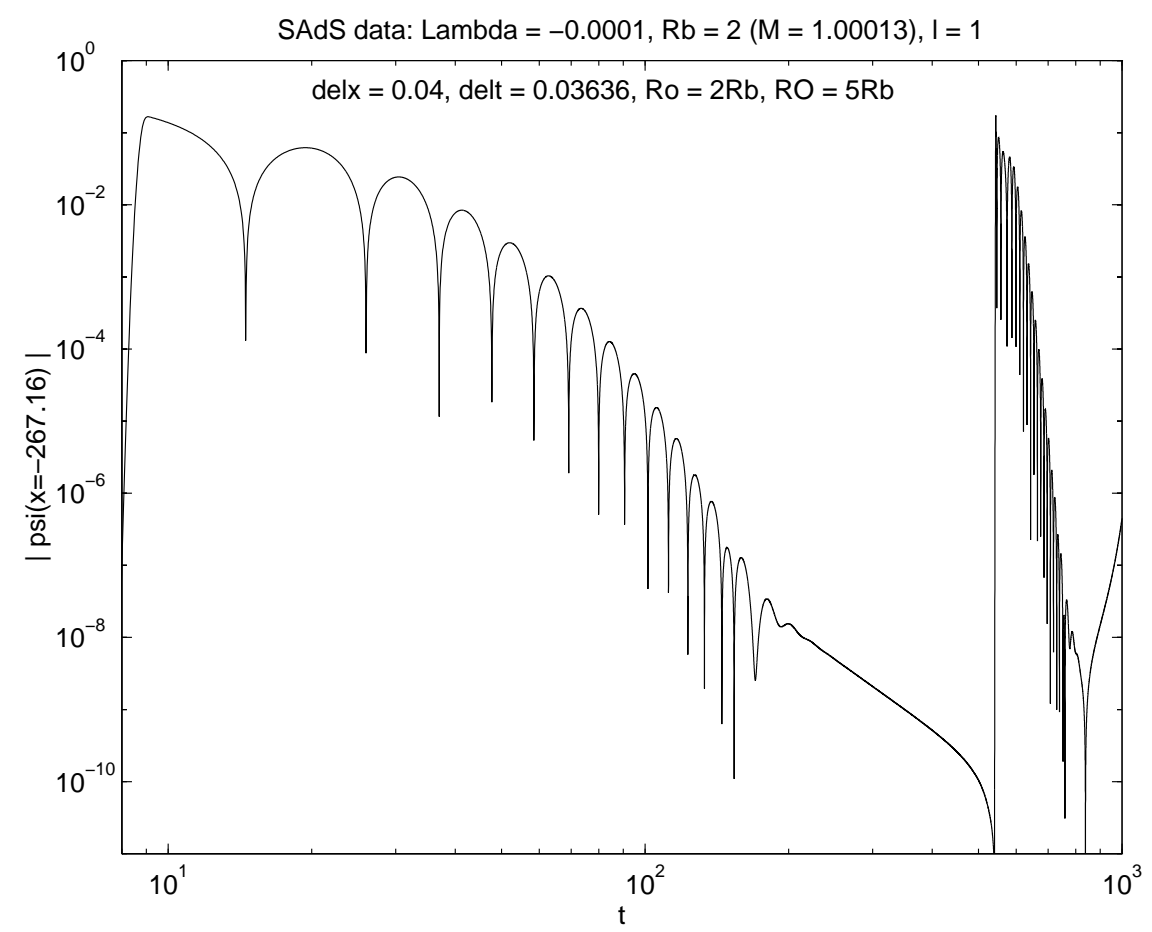

Figure 21: Loglog graph of the decay behaviour in SAdS background using $l=1$ and Dirichlet condition 


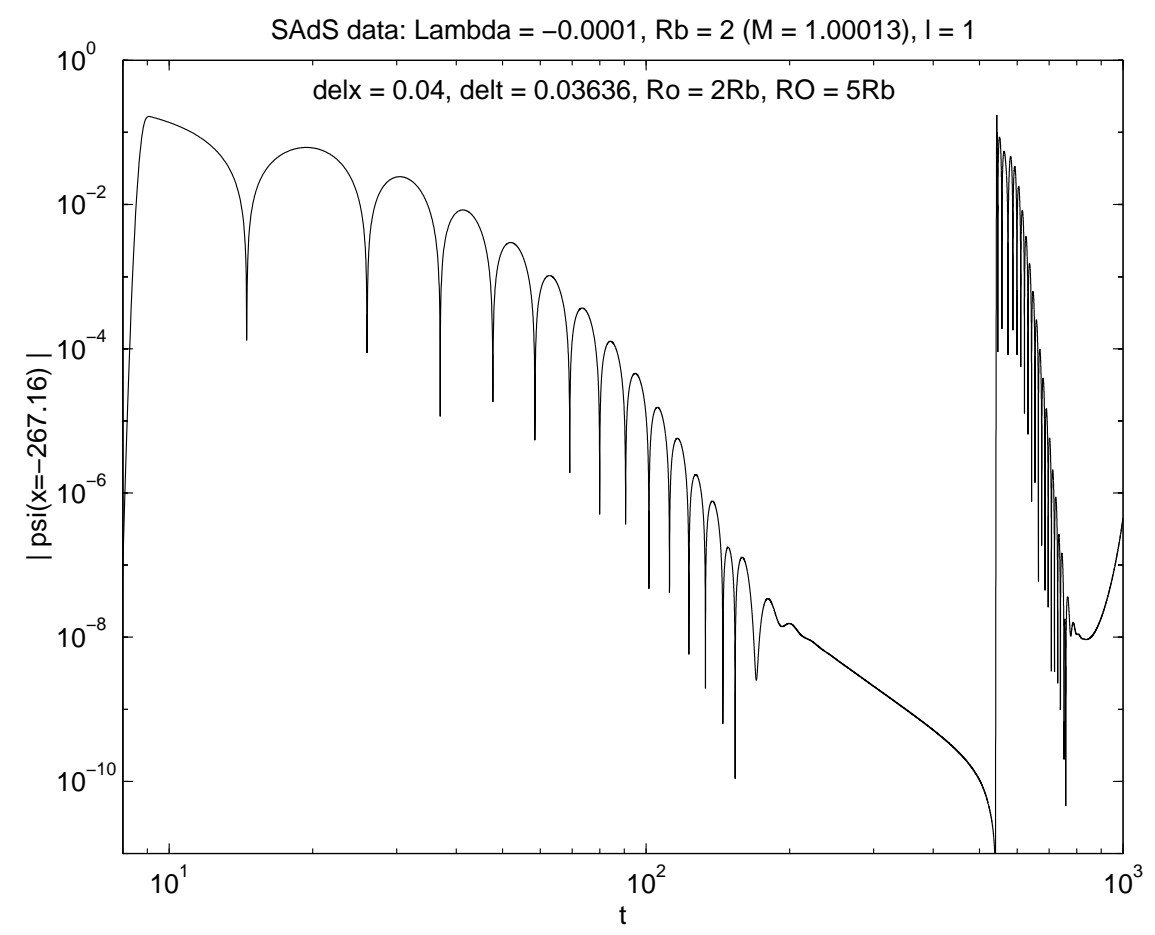

Figure 22: Similar graph to Figure 21 but using Neumann condition

Since the finite height of the potential maximum allows the scalar wave to surmount the barrier and leave the trapped region which is the exterior outside the barrier maximum, we expect that the peak value of the second returning wave is smaller than that of the first wave. However it is unclear from simple inspection of figures 19 to 22 whether or not this is the case. Figures 23 to 26 are the numerical results we obtained using the potential functions $(\mathrm{A})$ and $(\mathrm{C})$ in figure 18. We compute the large $|\Lambda|$ case with both Dirichlet (figures 23 and 25) and Neumann (24 and 26) boundary conditions. For this case there are more returning waves in a reasonable amount of CPU time. From these graphs, we can see that the scalar wave does indeed decrease but over a much larger time scale. Figures 23 and 24 indicate that the peak-height has an approximate exponential falloff for $l=0$. For $l>0$, the peak-height has a more complicated behaviour illustrated in figures 25 and 26. Over long time scales the maximum peak-height has a very mild approximate exponential falloff.

\section{Conclusion}

The results of this work indicate clearly that the asymptotics of a given spacetime have a considerable influence on the late time falloff behaviour of a scalar wave. The early work of Price showing that waves in Schwarzschild background decay away according to an inverse power rate was recently extended by Ching et al. who found that the inverse power-law can also be modified by a logarithmic term in some black hole configurations. We have been concerned in this paper with the falloff behaviour of conformal scalar waves in a background geometry which is asymptotically anti-de Sitter. We found that the waves in uncharged static and spinning 3D black hole backgrounds die out at an exponential rate. This conclusion is supported by both analytical and numerical computation. For conformal scalar waves in a Schwarzschild-anti-de Sitter background our numerical analysis demonstrates that the falloff pattern over short time scales obeys (after some ringing) a power-law falloff. However over longer time scales the outgoing wave returns from spatial infinity. The falloff of the peak amplitude in this case is neither inverse power nor exponential. However there 


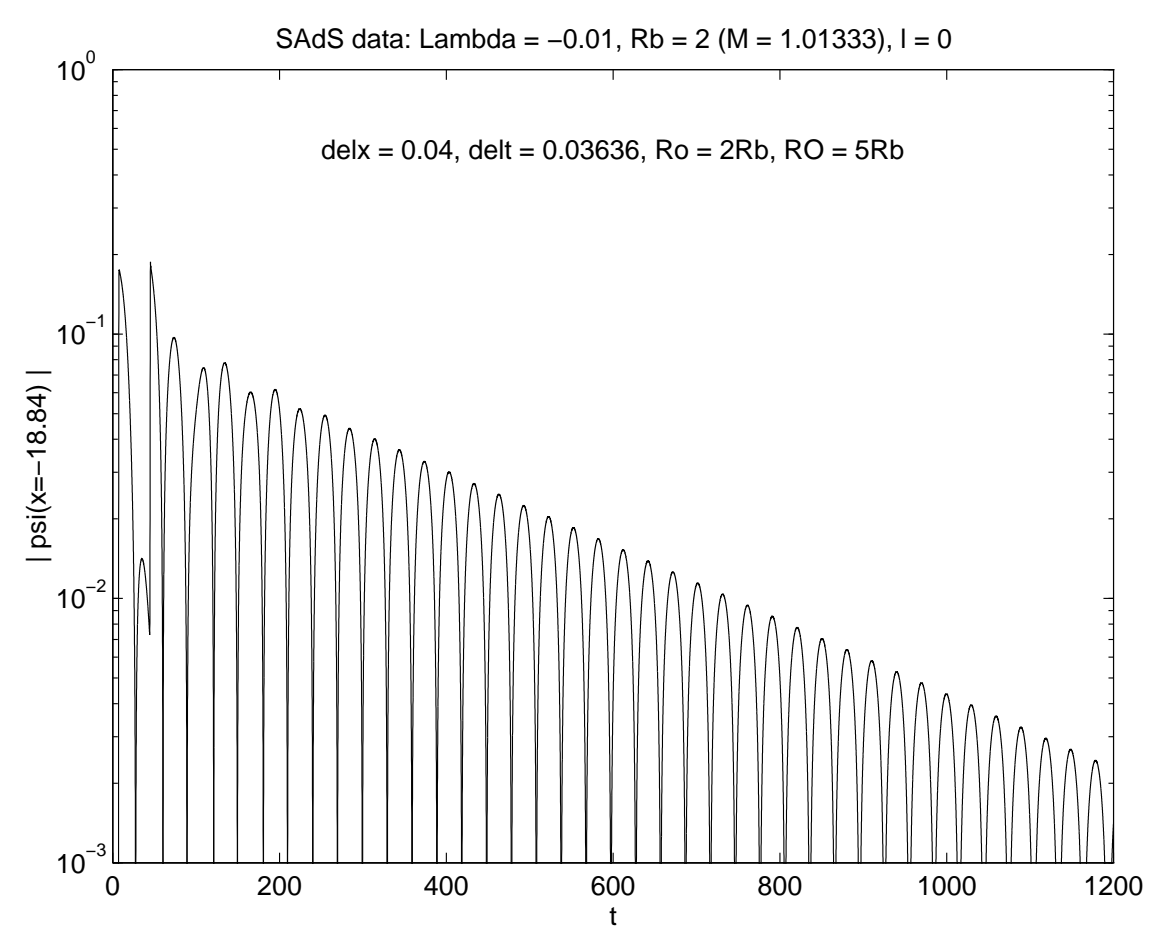

Figure 23: Semilog graph of the decay behaviour in SAdS background using $l=0$ and Dirichlet condition

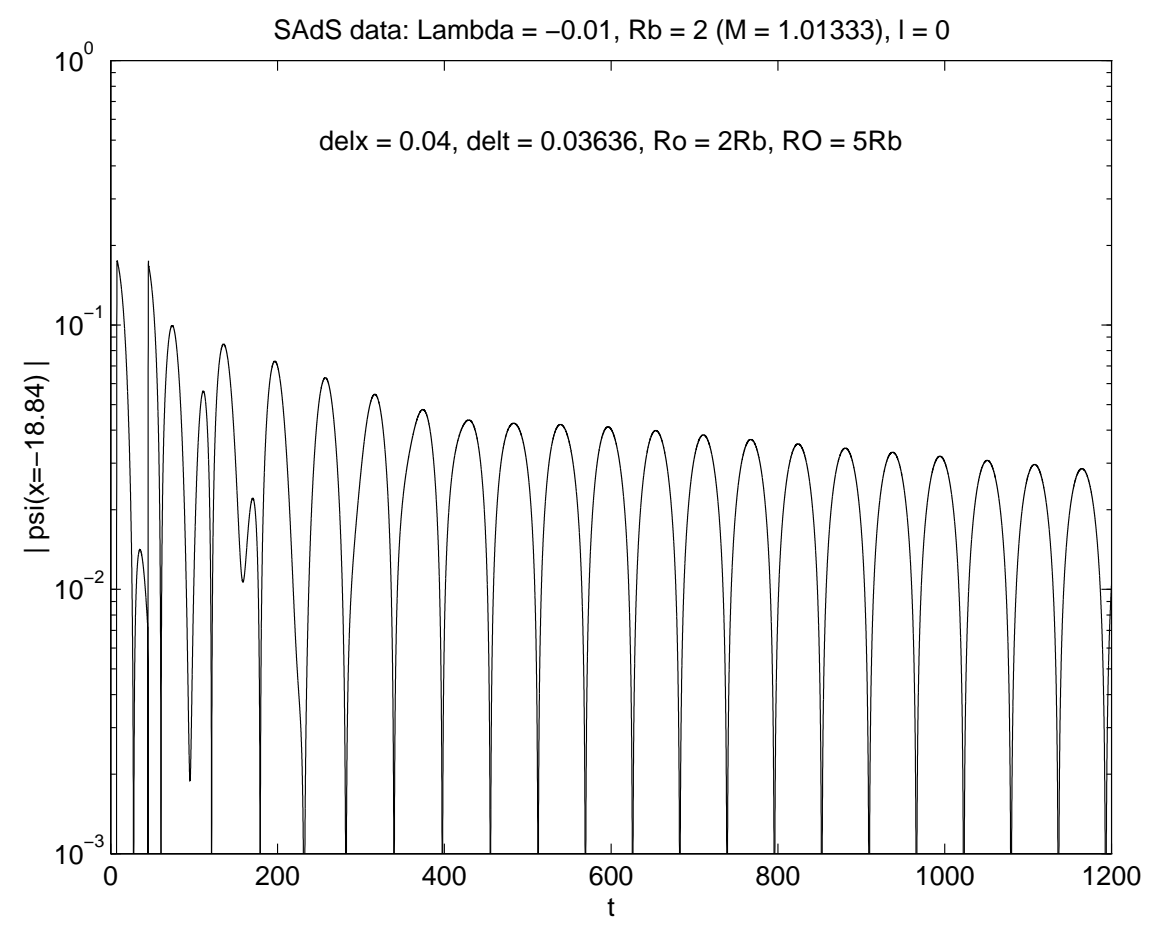

Figure 24: Conformal scalar wave decay behaviour using $l=0$ and Neumann condition 


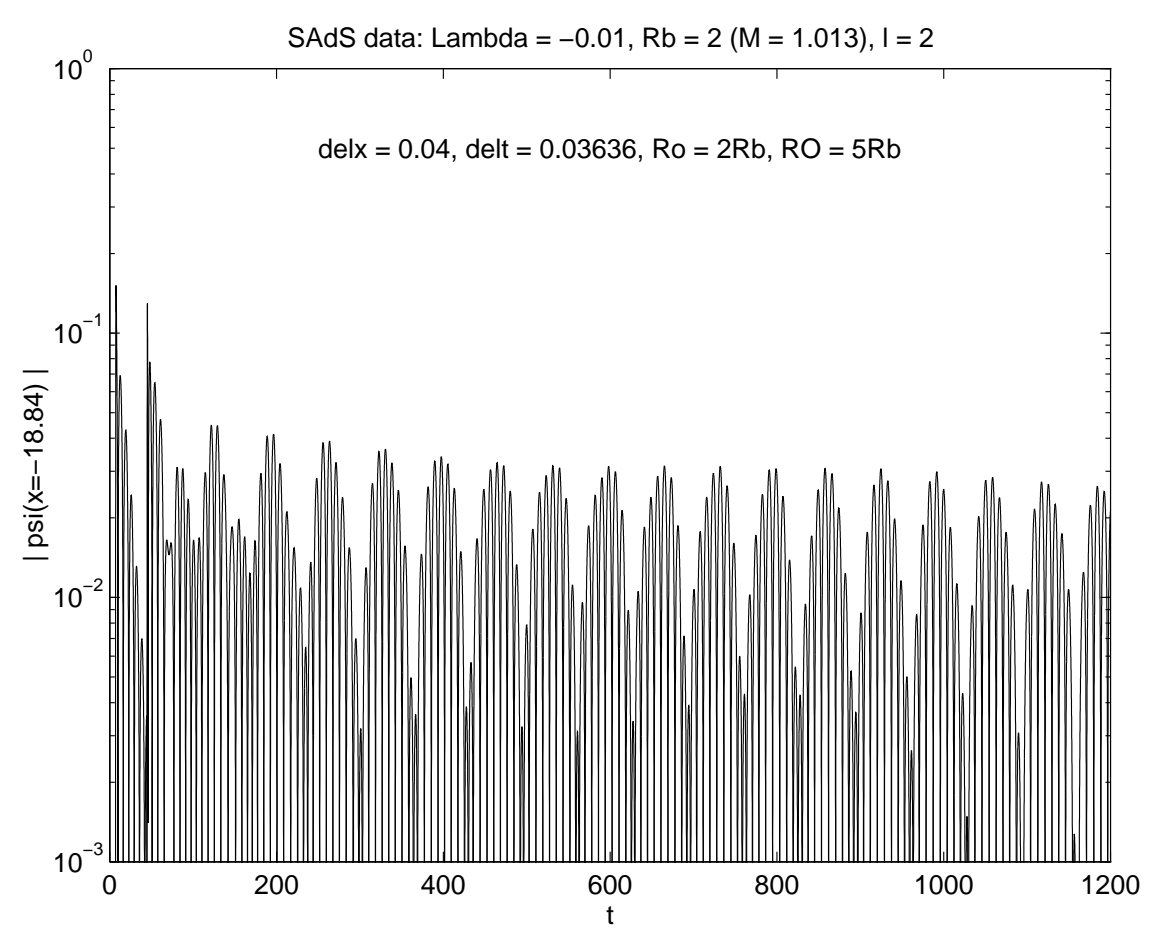

Figure 25: Semilog graph of the decay behaviour using non-zero $l$ and Dirichlet condition

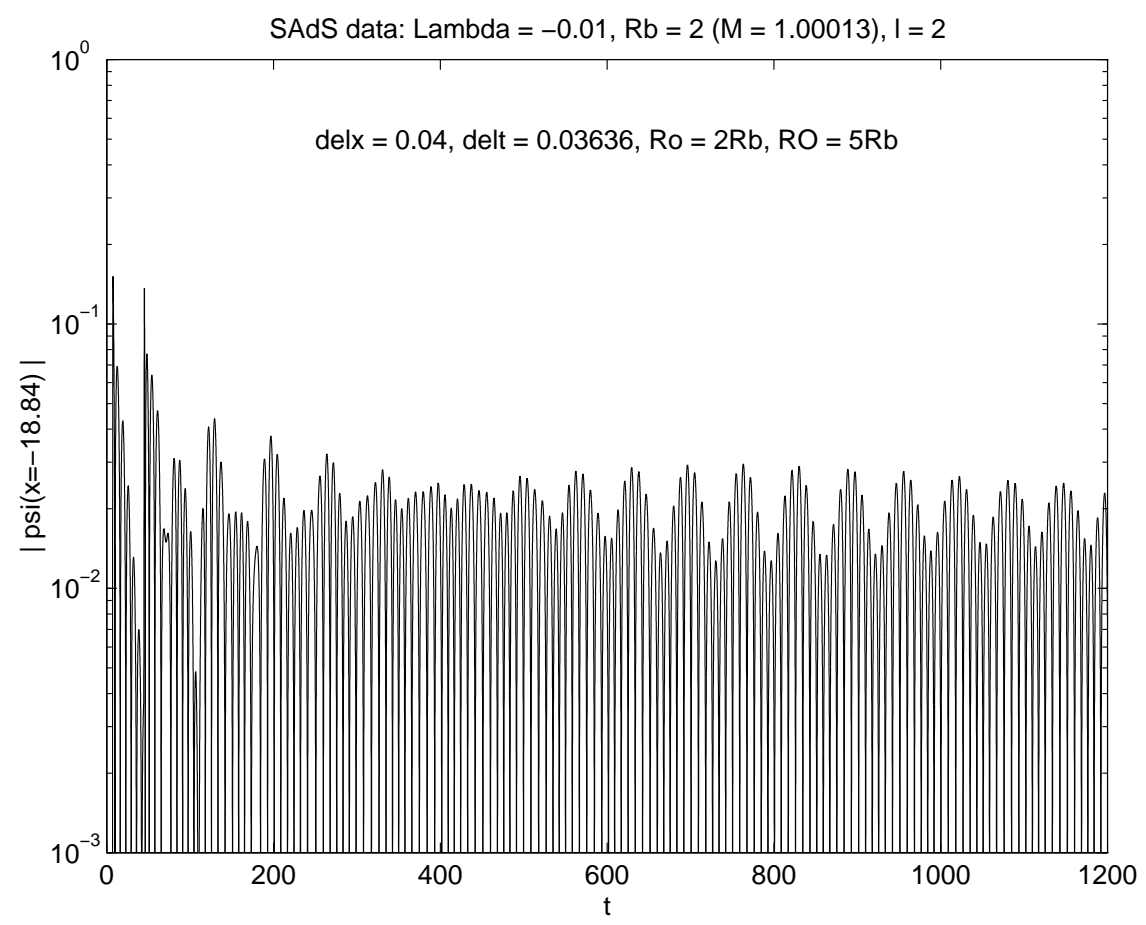

Figure 26: This semilog graph is the Neumann-analogue of Figure 25 


\begin{tabular}{|c|c|c|c|c|c|}
\hline$|\Lambda|$ & $M$ & $J$ & $|\Lambda| J^{2} / M^{2}$ & $\alpha$ & $\kappa$ \\
\hline 0.1 & 0.5 & 0.001 & $4 \times 10^{-7}$ & 0.3171 & 707.107 \\
\hline 0.1 & 0.1 & 0.001 & $10^{-5}$ & 0.1413 & 63.2452 \\
\hline 1.0 & 1.0 & 0.01 & $10^{-4}$ & 1.417 & 199.987 \\
\hline 0.1 & 0.01 & 0.001 & $10^{-3}$ & 0.04422 & 1.99875 \\
\hline 0.5 & 0.01 & 0.001 & $5 \times 10^{-3}$ & 0.09852 & 1.99374 \\
\hline 0.1 & 0.005 & 0.01 & 0.4 & 0.02034 & 0.0515936 \\
\hline 0.2 & 0.005 & 0.01 & 0.8 & 0.01541 & 0.0268999 \\
\hline
\end{tabular}

Table 1: Exponential falloff rate $\alpha$ which is found by graphical method is always less than the surface gravity $\kappa$ in rotating $3 \mathrm{D}$ black holes.

is a very weak exponential decrease in the maximal peak amplitude. This decay pattern is so complicated that further investigation will be needed to more precisely determine its dependence on $t$.

Previous investigations of mass inflation in a charged spinless 3D black hole 13 and an uncharged spinning 3D black hole [16] assumed a power-law falloff rate for the scalar wave. Under such assumptions these investigations demonstrated that mass inflation occurred in the corresponding geometries. We have shown here that the assumption of power-law falloff in the uncharged static and spinning 3D black hole background is not valid and must be replaced by an exponential falloff.

For a rotating 3D black hole we have shown that a $\phi$-independent scalar field also has late time exponential decay. In this particular setup, one can recalculate the mass inflation rate using the correct late time falloff rate. It is not difficult to show that the new inflation rate reads $m(v) \propto \exp ((\kappa-\alpha) v)$, where $\kappa$ is the surface gravity at the Cauchy horizon of the black hole. The parameter $\alpha$ comes from the exponential falloff rate for the scalar wave (which reads $\exp (-\alpha t)$ ) and is determined (say in the $J=0$ case) by setting $I_{\nu}\left(Z_{0}\right)=0$ for the relevant $Z_{0}$ and finding (numerically) the value of $\nu$ with the maximum negative real part. For example when $\Lambda=-0.1, M=0.01$ and $J=0.001$, the surface gravity at the Cauchy horizon is 1.999 but the parameter $\alpha$ is only 0.04 according to figure 17. Table 1 shows some other values of $\alpha$ and $\kappa$ in the rotating $3 \mathrm{D}$ black hole backgrounds. All these cases are consistent to the claim that the surface gravity $\kappa$ is always greater than the exponential falloff rate $\alpha$. Therefore the attenuation from the falloff effect does not halt mass inflation and the basic conclusions in [16 remain unchanged. Rather the mass inflation rate is slowed down relative to spacetimes with power-law falloff.

We have numerically determined that $\alpha$ is an increasing function of $l$. Recall that for the approximate potential $V(x)$ in (56), the dominant decay rate of the Green's function is determined by the root $\omega_{0}$ of the equation $I_{-i \omega / \lambda}\left(Z_{0}\right)=0$ in the lower half $\omega$-plane that is closest to the real axis. Since $Z_{0}=\sqrt{1+4 l^{2} / M}$, this root clearly depends upon $l$. We have computed this dependence and summarized the results in table 2 用 , where $\nu_{o} \equiv-i \omega_{o} / \lambda$. As $l$ increases (i.e. $Z_{0}$ increases), the real part of $-\nu_{o}$ also increases, yielding a more rapid falloff rate.

This leads to a qualitative distinction between the mass inflation mechanism in the (static) $(2+1)$ and $(3+1)$-dimensional spacetimes. In a Schwarzschild background, the falloff rate is $1 / t^{2 l+3}$ [9] which implies larger the moment $l$, faster the falloff. This yields a mass inflation rate $\sim \exp (\kappa v) / v^{2 l+3}[7]$; the exponential growth always surpasses the falloff effect for arbitrarily large $l$. For a 3D black hole with an inner horizon our results imply a falloff rate $\sim \exp ((\kappa-\alpha(l)) v)$. Therefore it is not difficult to imagine a situation in which $l$ is so large that the magnitude of the imaginary part of $\omega_{o}$ yields an $\alpha$ which exceeds the surface

${ }^{*}$ It is interesting to note that the roots with the $*$ in table 2 are far from precise. With only ten significant figures for $\nu_{o}$, the values of $I_{\nu_{o}}\left(Z_{0}\right)$ for $Z_{0}=20$ and $Z_{0}=30$ are of order $O\left(10^{2}\right)$ and $O\left(10^{9}\right)$ respectively. This is the way the modified Bessel function behaves for large $Z_{0}$. In the other cases, the corresponding values of $I_{\nu_{o}}\left(Z_{0}\right)$ are of order at most $O\left(10^{-5}\right)$. Indeed one will need more than 25 significant figures in order to bring $I_{\nu_{o}}(30)$ to the order $O\left(10^{-4}\right)$. 


\begin{tabular}{|c|c|}
\hline$Z_{0}$ & $\nu_{o} \equiv-i \omega_{o} / \lambda$ \\
\hline 1 & -1.296655185 \\
\hline 3 & $-2.246635543-1.620352348 i$ \\
\hline 5 & $-2.689085439-3.379067026 i$ \\
\hline 10 & $-3.416051394-7.973977837 i$ \\
\hline $20 *$ & $-4.325727136-17.46003536 i$ \\
\hline $30 *$ & $-4.961702418-27.09827714 i$ \\
\hline
\end{tabular}

Table 2: The roots for $I_{\nu_{o}}\left(Z_{0}\right)=0$ that are responsible for the dominant exponential falloff

gravity $\kappa$ which is independent of $l$ at the Cauchy horizon, cutting off mass inflation for those modes.

\section{Acknowledgements}

This work was supported in part by the Natural Sciences and Engineering Research Council of Canada.

\section{A Solutions in asymptotically flat $(D+1)$-dimensional back- ground}

Given the lapse function (23) in $(D+1)$-dimensional spacetime, the time-independent solution of $\mathcal{L}[\psi(r)]=0$ is given by equation (25). The coefficients $a_{j}(r)$ and $c_{j}(r)$ can be generated by the next two equations:

$$
\begin{aligned}
a_{j+1}(r)= & m(\ln |r|)^{\beta} a_{j}(r) \\
& -\frac{m[\xi(D-2-\alpha)(D-1-\alpha)+l(l+D-2-\alpha)]}{2 \gamma+1} W\left(1+(j+1) \alpha,(\ln |r|)^{\beta} a_{j}(r)\right) \\
& +\frac{m[\xi(D-2-\alpha)(D-1-\alpha)+(l+\alpha)(l+D-2)]}{2 \gamma+1} W\left(2 \gamma+2+(j+1) \alpha,(\ln |r|)^{\beta} a_{j}(r)\right) \\
& -\frac{m \beta[\xi(2 D-3-2 \alpha)+l]}{2 \gamma+1} W\left(1+(j+1) \alpha,(\ln |r|)^{\beta-1} a_{j}(r)\right) \\
& +\frac{m \beta[\xi(2 D-3-2 \alpha)-l-D+2]}{2 \gamma+1} W\left(2 \gamma+2+(j+1) \alpha,(\ln |r|)^{\beta-1} a_{j}(r)\right) \\
& -\frac{m \beta(\beta-1) \xi}{2 \gamma+1} W\left(1+(j+1) \alpha,(\ln |r|)^{\beta-2} a_{j}(r)\right) \\
& +\frac{m \beta(\beta-1) \xi}{2 \gamma+1} W\left(2 \gamma+2+(j+1) \alpha,(\ln |r|)^{\beta-2} a_{j}(r)\right) \\
c_{j+1}(r)= & m(\ln |r|)^{\beta} c_{j}(r) \\
& +\frac{m[\xi(D-2-\alpha)(D-1-\alpha)+(l+\alpha)(l+D-2)]}{2 \gamma+1} W\left(1+(j+1) \alpha,(\ln |r|)^{\beta} c_{j}(r)\right) \\
& -\frac{m[\xi(D-2-\alpha)(D-1-\alpha)+l(l+D-2-\alpha)]}{2 \gamma+1} W\left(-2 \gamma+(j+1) \alpha,(\ln |r|)^{\beta} c_{j}(r)\right) \\
& +\frac{m \beta[\xi(2 D-3-2 \alpha)-l-D+2]}{2 \gamma+1} W\left(1+(j+1) \alpha,(\ln |r|)^{\beta-1} c_{j}(r)\right) \\
& -\frac{m \beta[\xi(2 D-3-2 \alpha)+l]}{2 \gamma+1} W\left(-2 \gamma+(j+1) \alpha,(\ln |r|)^{\beta-1} c_{j}(r)\right)
\end{aligned}
$$




$$
\begin{aligned}
& +\frac{m \beta(\beta-1) \xi}{2 \gamma+1} W\left(1+(j+1) \alpha,(\ln |r|)^{\beta-2} c_{j}(r)\right) \\
& -\frac{m \beta(\beta-1) \xi}{2 \gamma+1} W\left(-2 \gamma+(j+1) \alpha,(\ln |r|)^{\beta-2} c_{j}(r)\right) .
\end{aligned}
$$

The function $W$ used above is defined as

$$
W(n, f(r)) \equiv \frac{r^{n}}{r} \int \frac{f(r)}{r^{n}} d r
$$

where $n$ is a real number and $f(r)$ is an integrable function. We evaluate the integral in $W$ in such a way that the integration constant is always set to zero. This function has a property that if $f(r)$ is a constant and $n \neq 1$, then $W(n, f(r))$ gives a constant. When $n$ is unity but $f(r)$ is still a constant, $W(n, f(r))$ is proportional to $\ln |r / \mathcal{R}|$, where $\mathcal{R}$ is some constant of dimension of length. In the case when $f(r)$ is no longer constant but a polynomial of $\ln |r / \mathcal{R}|$, the function $W$ gives another polynomial of $\ln |r / \mathcal{R}|$. The index $j$ in the two equations runs from zero to infinity and the starting coefficients $a_{0}(r)$ and $c_{0}(r)$ are two arbitrary constants. It is obvious that when the background is flat, i.e. $m=0$, all the $a_{j}(r)$ and $c_{j}(r)$ vanish except $a_{0}$ and $c_{0}$. If $m \neq 0$, these coefficients are polynomials in $\ln |r / \mathcal{R}|$.

For the equation $\mathcal{L}\left[C_{i+1}(r)\right]=2 d C_{i}(r) / d r$ with $\mathcal{L}\left[C_{0}(r)\right]=0$, the solution of $C_{i}(r)$ is given by

$$
C_{i}(r)=r^{\gamma+1+i} \sum_{j=0}^{\infty} \frac{c_{j}^{i}(r)}{r^{j \alpha}} .
$$

Each coefficient $c_{j}^{i}(r)$ can be calculated by the next two equations.

$$
\begin{aligned}
c_{0}^{i}(r)= & c_{0}^{i}=\frac{2^{i}(2 \gamma+1) !(\gamma+i) !}{\gamma !(i) !(2 \gamma+1+i) !} c_{0} \\
c_{j+1}^{i+1}(r)= & m(\ln |r|)^{\beta} c_{j}^{i+1}(r) \\
& +\frac{2}{2 \gamma+1}\left[\gamma W\left(-i+(j+1) \alpha, c_{j+1}^{i}(r)\right)+(\gamma+1) W\left(-2 \gamma-1-i+(j+1) \alpha, c_{j+1}^{i}(r)\right)\right] \\
& +\frac{m[\xi(D-2-\alpha)(D-1-\alpha)+(l+\alpha)(l+D-2)]}{2 \gamma+1} W\left(-i+(j+1) \alpha,(\ln |r|)^{\beta} c_{j}^{i+1}(r)\right) \\
& -\frac{m[\xi(D-2-\alpha)(D-1-\alpha)+l(l+D-2-\alpha)]}{2 \gamma+1} W\left(-2 \gamma-1-i+(j+1) \alpha,(\ln |r|)^{\beta} c_{j}^{i+1}(r)\right) \\
& +\frac{m \beta[\xi(2 D-3-2 \alpha)-l-D+2]}{2 \gamma+1} W\left(-i+(j+1) \alpha,(\ln |r|)^{\beta-1} c_{j}^{i+1}(r)\right) \\
& -\frac{m \beta[\xi(2 D-3-2 \alpha)+l]}{2 \gamma+1} W\left(-2 \gamma-1-i+(j+1) \alpha,(\ln |r|)^{\beta-1} c_{j}^{i+1}(r)\right) \\
& +\frac{m \beta(\beta-1) \xi}{2 \gamma+1} W\left(-i+(j+1) \alpha,(\ln |r|)^{\beta-2} c_{j}^{i+1}(r)\right) \\
& -\frac{m \beta(\beta-1) \xi}{2 \gamma+1} W\left(-2 \gamma-1-i+(j+1) \alpha,(\ln |r|)^{\beta-2} c_{j}^{i+1}(r)\right) .
\end{aligned}
$$

\section{References}

[1] S. W. Hawking and G. F. R. Ellis, The large scale structure of space-time, Cambridge: Cambridge University Press (1973).

[2] B. Carter, Phys. Rev. 174, 1559 (1968). 
[3] R. Penrose in Battlle Rencontres, ed. C. M. de Witt and J. A. Wheeler, New York: Benjamin (1968).

[4] M. Simpson and R. Penrose, Int. J. Theor. Phys. 7, 183 (1973).

[5] S. Chandrasekhar and J. B. Hartle, Proc. R. Soc. Lond. A 384, 301 (1982).

[6] W. A. Hiscock, Phys. Lett. A 83, 110 (1981).

[7] E. Poisson and W. Israel, Phys. Lett. B 233, 74 (1989); E. Poisson and W. Israel, Phys. Rev. D 41, 1796 (1990).

[8] A. Ori, Phys. Rev. Lett. 67, 789 (1991).

[9] R. H. Price, Phys. Rev. D 5, 2419 (1972).

[10] A. Ori, Phys. Rev. Lett. 68, 2117 (1992).

[11] S. Droz, Phys. Lett. A 191, 211 (1994).

[12] J. S. F. Chan and R. B. Mann, Class. Quantum Grav. 12, 351 (1995); Phys. Rev. D 51, 5428, (1995).

[13] V. Husain, Phys. Rev. D 50, R2361 (1994).

[14] R. G. Cai and D. F. Zhao, Phys. Lett. A 208, 281 (1995).

[15] A. Bonanno, Phys. Rev. D 53, 7373, (1996).

[16] J. S. F. Chan and R. B. Mann, Phys. Rev. D (to be published).

[17] F. Mellor and I. Moss, Phys. Rev. D 41, 403 (1990).

[18] E. S. C. Ching, P. T. Leung, W. M. Suen and K. Young, Phys. Rev. D 52, 2118 (1995).

[19] M. Banados, C. Teitelboim and J. Zanelli, Phys. Rev. Lett. 69, 1849 (1992); M. Banados, M. Henneaux, C. Teitelboim and J. Zanelli, Phys. Rev. D 48, 1506 (1993).

[20] F. J. Zerilli, Phys. Rev. D 2, 2141 (1970).

[21] G. Lifschytz and M. Ortiz, Phys. Rev. D 49, 1929 (1994); S. J. Avis, C. J. Isham and D. Storey, Phys. Rev. D 18, 3565 (1978).

[22] E. S. C. Ching, P. T. Leung, W. M. Suen and K. Young, Phys. Rev. Lett. 74, 2414 (1995).

[23] C. Gundlach, R. H. Price and J. Pullin, Phys. Rev. D 49, 883 (1994).

[24] J. D. Brown, J. D. E. Creighton, and R. B. Mann, Phys. Rev. D 506394 (1994).

[25] Handbook of mathematical functions : with formulas, graphs, and mathematical tables edited by M. Abramowitz and I. A. Stegun (Dover, New York, 1970). 